\title{
Towards the Elimination of White Spots in Mobile WiMAX Grids through Time Efficient Cognitive Vertical Handoff Algorithm
}

\author{
Abid Ali Minhas, ${ }^{1}$ Ahmed Qaddus, ${ }^{2}$ Muhammad Saeed Khan, ${ }^{3}$ Mohsin Iftikhar, \\ Tanveer Zia, ${ }^{4}$ Abdulaziz Almazyad, ${ }^{1,5}$ and Naveen Chilamkurti ${ }^{6}$ \\ ${ }^{1}$ Al Yamamah University, Riyadh 11512, Saudi Arabia \\ ${ }^{2}$ Wireless Research Center, Bahria University, Shangrila Road, Sector E-8, Islamabad, Pakistan \\ ${ }^{3}$ University College of Engineering, Sciences \& Technology, Lahore Leads University, Lahore, Pakistan \\ ${ }^{4}$ Charles Sturt University, Locked Bag 588, Boorooma Street, Wagga Wagga, NSW 2678, Australia \\ ${ }^{5}$ King Saud University, Riyadh 12372, Saudi Arabia \\ ${ }^{6}$ La Trobe University, Plenty Road and Kingsbury Drive, Melbourne, VIC 3086, Australia
}

Correspondence should be addressed to Mohsin Iftikhar; miftikhar@csu.edu.au

Received 2 October 2015; Accepted 29 December 2015

Academic Editor: Kamal Deep Singh

Copyright (C) 2016 Abid Ali Minhas et al. This is an open access article distributed under the Creative Commons Attribution License, which permits unrestricted use, distribution, and reproduction in any medium, provided the original work is properly cited.

During the past two decades particularly, there has been incredible growth in the telecommunication industry which evolved the demand for real time applications. Mobile grids and internet service providers are offering competitive real time services to customers in order to fulfil their daily demands. Nowadays, WiMAX has become a key player in telecommunication industry for providing cost effective communication solutions to customers residing in developed and underdeveloped regions. Moreover, a significant role of WiMAX technology is to fill the gap between the first-world and third-world countries. WiMAX technology offers affordable low cost wireless multimedia services to its customers by using point to multipoint propagation methods. Over the past few years, the problem of white spots in WiMAX grids has been reported in the literature extensively. Consequently, this issue has got a lot of attention from researchers. In order to disentangle the phenomenon of white spots, a novel algorithm based on time efficient cognitive vertical handoff scheme has been presented in this paper. The proposed algorithm is based upon principles of cognitive heterogeneous wireless grids. The simulation results have shown that the proposed algorithm is a promising candidate to overcome the problem of white spots in mobile WiMAX grids.

\section{Introduction}

The WiMAX radio frequency technology for Wireless Metropolitan Area Network (WMAN) grid is based upon principle of IEEE 802.16-2004 standard [1]. WiMAX IEEE 802.162004 standard has become a good and affordable alternative against fixed lines wired grid like Public Switched Telephone Network (PSTN) and is an attractive replacement for cord and Digital Subscriber Line (DSL) utilities. WiMAX IEEE 802.16e standard is capable of providing association among static Base Transceiver Stations (BTS) along with mobile gadgets. High speed handoff is feasible for end users located in rapidly moving transport by using IEEE 802.16 e standard, which entirely holds the mobility of mobile users [1-3].

Through practicing Orthogonal Frequency Division Multiple Access (OFDMA), WiMAX can accommodate multipath execution for near line of sight intercommunication. One enhanced feature of WiMAX IEEE 802.16e standard endures Scalable Orthogonal Frequency Division Multiple Access (SOFDMA), which basically restricts the interference between different end users and can accommodate high data rate applications and enhances scalability as well. Quality 
of Service (QoS) and security are the two fundamental requirements in WiMAX grid. WiMAX can provide pervasive, point to point, and point to multipoint broadcasting by using flexile engineering and better encryption methods. WiMAX is very flexible in terms of requiring less time for the deployment of the grid. Moreover, WiMAX is capable of providing interoperability between different vendor's devices and portability with existing grids. The usage of higher modulation and channel bandwidth methods allow WiMAX to accommodate its users with high capacity [2-7].

\section{Background of WiMAX}

WiMAX forum has proposed different WiMAX standards. One of the key features of initial WiMAX standard IEEE $802.16 \mathrm{a}$ is that it can be executed in licensed and unlicensed frequency bands and has a frequency band spectrum range from 2 to $11 \mathrm{GHz}$. In IEEE 802.16a WiMAX standard transceiver antennas of source and destination devices do not require direct line of sight between each other as the devices operate in low frequency ranges. WiMAX forum introduced IEEE 802.16 b standard for providing good quality to real time voice and data services customers. It has operating frequency band range from 5 to $6 \mathrm{GHz}$. WiMAX IEEE 802.16c standard allows interoperability between different vendor devices and gadgets and has an operating frequency band range from 10 to $66 \mathrm{GHz}[3,8]$.

For compliance testing amongst different vendor devices, WiMAX forum proposed much improved and well equipped version of IEEE 802.16a standard known as IEEE 802.16d. Lastly, IEEE 802.16 e standard by WiMAX forum suggests nonline of sight communication in a wider broadcasting area. It also provides the capability to mobile users to communicate moving at vehicular speeds [3].

"White spots" are basically the operation situated in the WiMAX grid broadcasting that has nil broadcasting from the serving base station(s) due to undefined and unexpected events. In the literature white spots are also named as blind spots [9], black spots [10], and hot spots [11] where grid broadcasting is not available to WiMAX subscribers. WiMAX broadcasting area cannot be marked with exact certainty on the topographical depiction as white spots are not precisely located on the atlas. Two primary ways for surveying the broadcasting region of a WiMAX grid are presented in subsequent sections [3].

2.1. Empirical Model. Empirical model is the first model that supports the principle of trial based installation and is equipped with empirical measurements. Statistical tools used by empirical model are used to perform in-depth analysis of data rate of a particular broadcasting area of a WiMAX grid. The models based on easy principles of empirical modeling fail to detect white spots in the WiMAX broadcasting area. The principle of statistical probability is followed by empirical modeling which fails to provide accuracy for WiMAX base station sites. Hence empirical modeling fails to provide the appropriate link budget plan for the commissioning of WiMAX base stations $[3,12]$.
2.2. Site Specific Model. The second type of model is known as site specific model and is based on deterministic methods. The broadcasting area of WiMAX grid is analyzed by software tools. This model is able to detect the exact position of the target on the atlas by using tools like Global Positioning System (GPS). Further, site specific model does not follow the theory of probability and uses software. But, the hurdles located in topographical region cannot be traced by this site specific model, which is the potential source of communication obstruction. The interruption is due to the influence of multipath and fading signals received at the destination which is caused by the reflection of signals from the obstacles present in their path. If the transmitted direct signal and the obstructed signal reach the destination at the same time, then both signals will cancel each other due to 180-degree phase shift. Ultimately, due to cancellation of transmitted signal and obstructed signal, the destination will receive no signal. Software maps based on GPS are used to gather information about dry and wet areas of topographical regions. The ray tracing or ray launching algorithms used in deterministic software model are supported by homogeneous geometrical theory of diffraction. The broadcasting spectrum of WiMAX differs from other wireless technologies with respect to its equipped features. In near line of sight intercommunication, the performance and result accuracy of site specific or deterministic software model are much better than the statistical empirical model which is based on calculations derived from Maxwell's theory. Hence under this contemporary condition, the site specific or deterministic software model has the upper hand in terms of more accurate results as compared to empirical or statistical model $[3,12]$.

Figure 1 has two parts, where part (a) shows a general broadcasting model [12] and part (b) shows a specialized WiMAX broadcasting model [12]. White spots are not present in the simple broadcasting area which is also referred to as a general broadcasting model. White spots are present in WiMAX grid broadcasting area. It was practically proven by telecom hardware equipment vendors through commissioning and measuring the Received Signal Strength Indicator (RSSI) of single base station in WiMAX grid. This illustrates that the WiMAX technology can be used during line of sight (LoS) communication in Metropolitan Area Network (MAN) grids deployed in cities but it is not feasible for near line of sight (NLoS) communication. During testing some noncoverage network area (NLoS WiMAX grid broadcasting area commissioned in thickly inhabited urban surroundings), some white spots were reported due to obstructions and obstacles present in the path between the transmitting and receiving station as shown in Figure 1(b) $[3,12]$.

During the test an omnidirectional antenna equipped with spectrum analyzer was utilized at destination site. Coverage up to $30 \mathrm{~km}$ can be obtained in LoS scenario for reliable communication. Coverage up to $5 \mathrm{~km}$ can be obtained in NLoS scenario. In the event of testing NLoS communication mode, not all of the receivers present in the WiMAX base station coverage area were receiving transmissions. White spots in the WiMAX broadcasting area can be identified from these noncoverage areas or empty spots, where base station RSSI has not been received at the WiMAX receivers. 


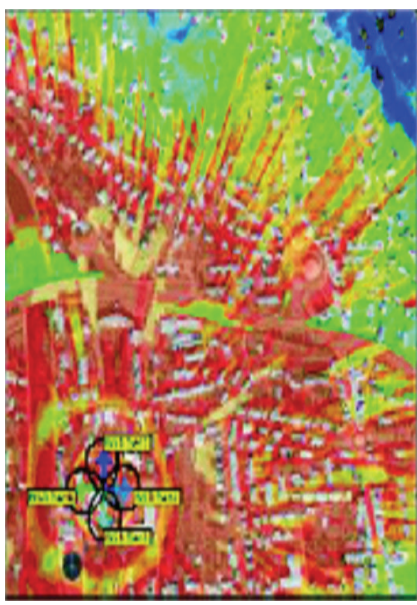

(a)

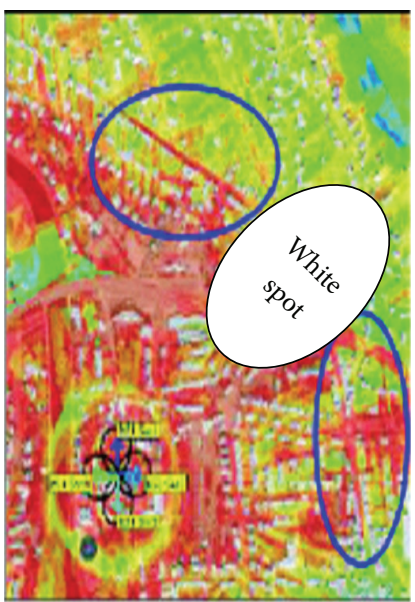

(b)

Figure 1: WiMAX Coverage Model [12].

After recording these practical results it was noted that the RSSI values being calculated on the basis of theoretical deterministic software models differ from the actual obtained results. Hence the outcome of this trial scenario proved that both empirical and deterministic theoretical models actually failed to calculate accurate RSSI values for link budget design used for commissioning WiMAX sites. Different obstructions and hurdles like skyscrapers, advertisement boards and vegetated areas, and so forth are the major sources of reflection which result in weaker or no signal availability at some spaces in the WiMAX broadcasting region. The obstructions between the base station and the destination result in poor broadcasting due to lack of signal strength. Even though the user is using enhanced modulation schemes for data transmission still a minor movement between the user and the serving base station can result in signal degradation. Due to the hurdles like white spots present in the WiMAX broadcasting spectrum, it is quite complex to design a network based on measurements obtained from software or deterministic models. The crosses in Figure 2 represent white spots in the broadcasting area of the WiMAX spectrum $[3,12]$.

In order to have uninterrupted communication between the base station and the subscriber station and to avoid white spots in WiMAX grid, the WiMAX operator has to increase capital expenditure for network deployment. White spot can be a potential problem and can even lead to call drop in the event of handoff procedure when the WiMAX subscriber is roaming and is shifting from one base station to another to have an established and uninterrupted connectivity with WiMAX base station. The fast movement of WiMAX mobile user at vehicular speed is supported by the WiMAX 802.16e standard. When the WiMAX mobile user is moving at vehicular speed, there is a potential risk of disconnectivity from WiMAX base station. It is very challenging to reveal the white spots or no signal areas present in the WiMAX broadcasting spectrum. Also, in order to prevent the white spots, it is quite

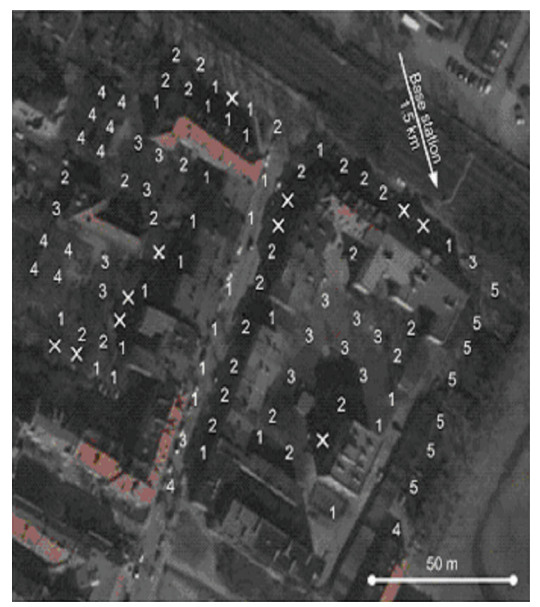

Figure 2: WiMAX coverage [12].

complex to calculate the exact number of required base stations to cover a specific WiMAX broadcasting area through using some theoretical network planning tools $[3,12]$.

The propagation of WiMAX broadcasting range exists differently for indoor, urban, and rural environments. The broadcasting spectrum encounters different types of obstacles which are the main cause of the signal loss or fading in WiMAX broadcasting area. The propagation models being used have limited information regarding the objects present on the map. For example, in case of an urban environment, the propagation model only displays the $3 \mathrm{D}$ image of the buildings. The height of the building is set by default, which could differ from the original height of the building. This propagation model does not specify the obstacles or obstructions like trees, billboards, and towers, which can affect the WiMAX broadcasting area. For designing an indoor environment, it is essential to plan the number and specific 
size of windows and doors present in the building. Further, it is very difficult to combine the two databases of urban and indoor environment because of the limited memory of standard PC systems $[3,13]$.

In an indoor environment, the main causes of signal loss and diffraction are the horizontal walls and the vertical roofs. The empirical models based on statistical formulas are also used for WiMAX grid planning. This mainly depends upon the direct LoS between the transceiver equipment pieces. In metropolitan environment, the direct beam consists of high rooftop scattering whereas in the indoor environment the direct ray includes direct line between the transmitter and the receiver. In both cases, direct ray cannot be achieved between the transmitter and the receiver due to the obstructions, obstacles, and attenuation. Another WiMAX grid planning model is ray optical model. This requires high computation and processing time. In most of the cases, $98 \%$ of energy is acquired by two to three rays only for communication between transmitter and receiver. The rest of the rays are lost due to obstacles, obstructions, and attenuation between the transmitter and receiver. Hence the nonsignal broadcasting area (i.e., also referred to as white spots in the WiMAX grid broadcasting area) cannot be detected by ray optical model $[3,13,14]$.

\section{Related Work}

Here, we describe the related work on white spots in WiMAX grids in detail. We have explained earlier the limitations of two popular types of WiMAX propagation (i.e., empirical and site specific) models. The empirical models which basically rely on statistical tools are used to analyze the broadcasting area for WiMAX grid. But, unfortunately, the white spots present in the WiMAX grid broadcasting area cannot be detected by empirical models. For tracing locations on the global map, site specific models, which mainly depend upon specialized propagation tools like Global Positioning System (GPS), are used. But the site specific model can only find or trace the location on the map. It is unable to provide the evidence regarding obstacles or obstructions present on the earth surface, which results in white spots and further stimulates the call drop or communication disruption for an active call $[3,12]$.

The problem of white spots in WiMAX grid has been addressed by few researchers. One proposed solution to avoid white spots has been presented by Gierlowski et al. [12]. They have proposed that the problem of white spots can be avoided by using a homogeneous approach based on mesh topology and commissioning large number of base stations. But by doing so, the capital expenditure and operational expenses will increase hysterically, consequently making this solution nonpractical.

The blind spots also referred to as white spots can be removed by multipath cellular architecture as discussed by $\mathrm{Al}$ Yusuf and Murshed in [9]. In the suggested idea, a mobile station while communicating with the wireless network base station via a single base station should also be connected to two other base stations. Hence, in the event of appearing blind spot, the mobile station can initiate handoff to the remaining base station present in its range and avoid disconnectivity from wireless network. Under these circumstances, a large number of base stations should be commissioned in a multipath cellular architecture, which is not very suitable to be used in WiMAX grid.

Zaggoulos et al. [15] have proposed that modulation algorithms which basically rely on adaptive channel modulation schemes can be used to eliminate the problem of white spots. In their analysis, the authors have compared the simulation and experimental results obtained for downlink Packet Error Rate (PER). It has been reported that results of soft and hard implementation of the proposed idea significantly differ from each other. According to authors, it is not possible to eliminate the white spots present in wireless broadcasting areas even with the use of well enhanced modulation algorithms in real time scenario. Chadwani et al. have argued about the use of relay stations [10] in wireless grids to eliminate the problem of black/white spots present in the broadcasting area of wireless grids for performance enhancement. For the transmission of data from source to destination, the authors have proposed a solution in the form of relay stations for relaying information from source to destination. The use of fixed relay stations is necessary for increasing cell capacity and improving the base station coverage region. Through the usage of fixed relay station, the battery life of the subscriber mobile station and power required by the mobile user during transmission can be saved. Relay stations cannot be used to eliminate the white spots in wireless network broadcasting area because they can only function as a booster or amplifier. It has been noted that relay station can only be used to increase the capability of the system but fails to increase the broadcasting area of the wireless grid.

The utilization of relay stations and repeaters has been discussed by Davydov et al. [16]. Again through using relay stations, the broadcasting area of the wireless grid cannot be increased; it can only be used to increase the capability of the system in real time scenario. The problem of noise and interference has been observed when a large number of repeaters are being commissioned in the broadcasting area of a single base station. Consequently, this results in decreasing the broadcasting area of serving base station.

The commissioning of distributed wireless sensor nodes with fixed battery source to eliminate white spots has been proposed by Jaichandran et al. [11]. By deploying wireless sensor grid nodes, we can reduce the white spots and can extend the life of the grid but for the time being only. Consequently, the commissioning of wireless sensor grid nodes is not a feasible and long term solution as it still fails to trace the white spots in the wireless grid broadcasting region. Another important point in general to be noted is that wireless sensor nodes are battery operated and are having very small data rate due to which the proposed solution is not very feasible.

Kan et al. in [17] have presented a solution according to which the deployment of dual channel scheme could be feasible to avoid white spots in WiMAX grid. The proposed idea is as follows. Due to the obstructions in grid broadcasting area, when blind/white spots appear, GPS technology will be used to provide location estimation services to the mobile 
users through utilizing dual channel positioning systems. It can help to avoid blind spots in grid broadcasting area. But the main drawback of GPS technology is that it is not available to mobile users when they are present in an underground road, underpass, railway tunnel, or the basement of a building. Hence again, the deployment of dual channel scheme, which is basically founded in GSM grids and GPS technology, cannot eliminate blind spots in a wireless broadcasting area. Further, since GPS is based on satellite technology, which is often affected by weather storms and can cause complete telecommunication blackout on earth, the idea of using dual channel positioning system for avoiding blind spots in GSM grid broadcasting area is also not technically very efficient.

$\mathrm{Lu}$ and $\mathrm{Wu}$ have proposed the idea of using two-hop relay stations [18] in cellular grids to improve the performance and to remove the dilemma of dead spots or white spots present in the broadcasting area of cellular grids. The usage of two-hop relay stations might be useful for increasing capacity of cell and for the enhancement of broadcasting area of cell. Two-hop relay stations can be used to eliminate the white spots located in cellular grid broadcasting region but this will increase the capital cost of the communication system. Another point is that relay station will be acting as an amplifier or booster. Two-hop relay stations may increase the capability of cellular grid but fail to increase broadcasting region of cellular grid. Thus the method proposed here is not cost effective $[18,19]$.

The problem of blind spots in wireless sensor grids has also been addressed by Jin et al. [20]. Their proposed solution is based on Voronoi graph which basically relies on statistical formulas. Voronoi graph is used to locate blind spots, which are calculated by different wireless sensor nodes present in the wireless grid broadcasting area. The Voronoi graph analyzes the data of the wireless sensor grid broadcasting area by using statistical tools. The statistical techniques like Voronoi graph when used in real time scenario actually fail to detect blind spots in the wireless sensor grid broadcasting area due to their probabilistic approach.

\section{Problem Statement}

In WiMAX grid broadcasting region, call drop occurs due to obstacles, obstructions, and hurdles present in the path of WiMAX mobile user and WiMAX base station. These regions of nonsignal coverage areas are also referred to as white spots which is the major reason behind weak broadcasting of WiMAX base station. The primary motive for conducting this research is to propose a practical, efficient, and cost effective solution to avoid nonsignal coverage regions, that is, white spots in WiMAX grid broadcasting region. The proposed solution will be able to provide uninterruptible connectivity from mobile subscriber to the base station.

\section{Proposed Model}

White spots present in WiMAX broadcasting region cannot be eliminated through the utilization of WiMAX mesh architecture, so to counter this dilemma, an innovative scheme based on cognitive heterogeneous wireless grid is proposed.
Cognitive heterogeneous approach has been adopted in this work to utilize the existing GSM services. To continuously access the real time applications such as voice, video, and data in a mobility scenario, the WiMAX mobile subscriber holding a cognitive mobile device will be exploiting a heterogeneous scheme. Through utilizing the heterogeneous approach, vertical handoff and switching towards GSM grid will be automatically triggered by cognitive mobile subscriber without any intervention or delay in the scenario of white spots, where the WiMAX grid is not available to accommodate the continuity of the call. Hence the dilemma of disconnection or call cutoff can be avoided by using heterogeneous approach. The signal loss will occur differentially with respect to time when the user will enter in the domain of white spots. Hence, we can say that when the WiMAX subscriber is entering in the nonsignal coverage regions, that is, white spots, there will be no sudden transform in the subscriber's Received Signal Strength (RSS). The Mobile Unit (MU) continuously stores the information about RSS collected at Forward Channel (FC) in its buffer during the event of decrease in RSS with respect to time. For certain optimized interval of time it will find the first derivative $d(\mathrm{RSS}) / d t$. The decision for horizontal or vertical handoff is taken on the basis of calculated result of second derivate $d^{2}(\mathrm{RSS}) / d t^{2}$. In the proposed design, priority is given to horizontal handoff. When the problem of white spot occurs, the WiMAX MU hunt for alternative WiMAX base station present in the nearby vicinity is initiated. If WiMAX MU succeeds in its hunt, then horizontal handoff procedure is executed and MU is handed over to WiMAX BS present in the nearby vicinity. If RSS is approaching critical value, then MU switches to GSM grid and vertical handoff is initiated. The following subsection contains the detail about it.

5.1. Grid Selection Criteria. The grid selection is performed on the basis of RSSI levels and required Quality of Service (QoS). When the RSSI levels at the WiMAX mobile subscriber drop below the desired threshold limit for maintaining connectivity to base station, then the selection of handoff from one type of grid to another type of grid is initiated. In this study the authors have tried to utilize the existing GSM services $[9,12]$ instead of commissioning the new base station. Hence the projected strategy is more effectual in terms of cost than its predecessors. Figure 3 shows the incorporation between GSM/WiMAX grid and 802.21 information server backhaul connectivity [21].

The main improvement of projected approach is that it does not rely on the principles of point to multipoint technique as in case of homogenous method [22] where all operations of the grid activity are managed and apprehended by a single base station. We can utilize homogenous method efficiently to eliminate white spots, when there are a large number of base stations commissioned in a specific broadcasting area. The capital expenditure of the WiMAX mesh grid will significantly multiply when a large number of base stations are commissioned in a specific broadcasting area. Thus WiMAX mesh architecture is not viable to be commissioned in a metropolitan area grid [12, 21].

The technologies such as WiMAX and GSM being utilized in the proposed method support the mobile subscriber 


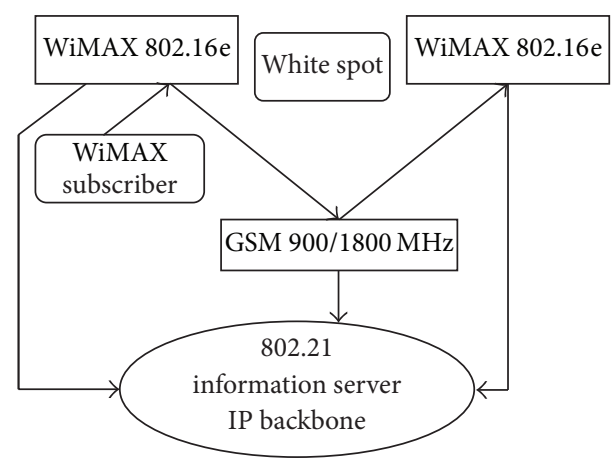

FIGURE 3: Cognitive heterogeneous approach of proposed model.

when present in a vehicle moving at $140 \mathrm{~km} / \mathrm{hr}$. During the scenario of horizontal handoff when a neighboring WiMAX base station is not present in the nearby vicinity of WiMAX grid, then vertical handoff is initiated to another wireless grid present in the area, which avoids the odds of call drop [21].

To disentangle the dilemma of white spots in WiMAX grid broadcasting area, a projected model based on cognitive heterogeneous wireless grid approach is depicted in Figure 3. The vertical handoff scenario of mobile subscriber from WiMAX base station to GSM base station and vice versa during the development of white spot has been elaborated with the help of flowchart in Figure 4 [3].

Figure 5 shows the vertical handoff scheme from WiMAX to GSM grids and vice versa along with customized edition of IEEE 802.21 protocols which is most suitable to our research approach. The handoff procedure of mobile WiMAX when used in heterogeneous grid is depicted in Figure 5. Initially, the WiMAX mobile user is connected to the WiMAX grid and is accessing voice and data services from the WiMAX grid. The RSSI value starts to fall below the desire threshold value required for maintaining connectivity between the WiMAX mobile station and WiMAX base station as the white spot crops up. Now handoff is required for avoiding communication disconnectivity, so the 802.21 information server will search for the availability of the neighboring Public Land Mobile Grid (PMLN) by sending MIH_GET_Information.request to 802.21 information server. Now when the PLMN is located (which in this case is GSM grid), the 802.21 information server will send MIH_Link_Detected message to mobile station. On receiving MIH_Link_Detected message, the mobile station will now send MIH_Candidate_Query.request to 802.21 information server for handoff to GSM grid base station. Now 802.21 information server will send MIH_Candidate_Query.confirm to mobile station which confirms that resources are available for handoff to GSM base station. Now mobile station will initiate handoff to GSM base station by sending MIH_Handover_Commit command. When handoff to GSM base station is completed, GSM base station will be acknowledged by sending MIH_Link_Handover_Complete command to mobile station [21, 23].

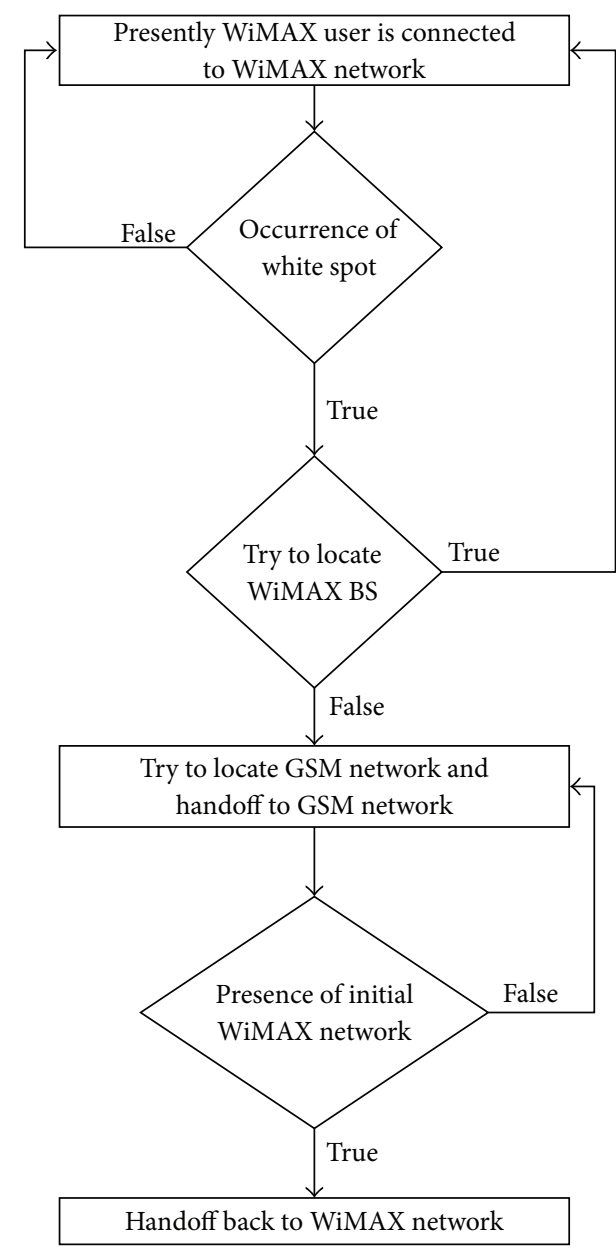

FIGURE 4: Flowchart of network selection.

During this alternative connectivity between the WiMAX mobile station and GSM grid, the mobile station also searches for the availability of the previous initial WiMAX grid by listening to MIH_Link_PDU_Transmit_ Status, which is repeatedly broadcast by 802.21 information server. If previous (initial) WiMAX grid becomes available, then the WiMAX mobile station will first check the RSS value by MIH_Link_Detected. If the RSS value is above the minimum threshold level required for maintaining connectivity between the mobile station and WiMAX base station, then the mobile station will follow the same previous step of sending MIH_Candidate_Query.request to IEEE 802.21 information server [7, 21, 23]. Now 802.21 information server will send MIH_Candidate_Query.confirm to mobile station which confirms that resources are available for handoff back to initial WiMAX base station. Now mobile station will initiate handoff to WiMAX base station by sending MIH_Handover_Commit command. When handoff to WiMAX base station is completed, then WiMAX base station will be acknowledged by sending MIH_Link_Handover_Complete command to mobile station $[21,23]$. 


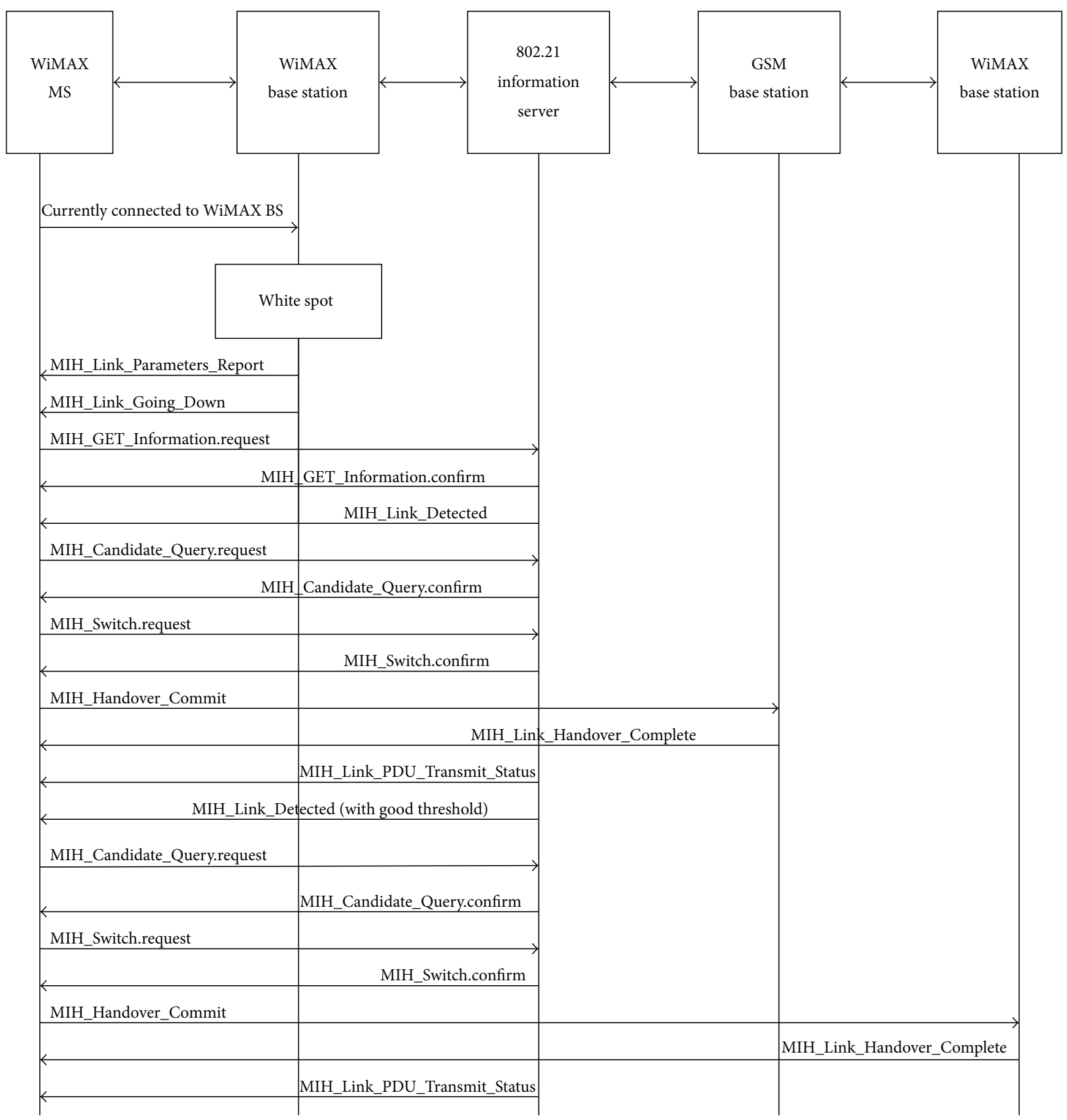

FIGURE 5: Cognitive mobile handoff procedure.

5.2. Handoff between WiMAX and GSM Grids. Whenever a handoff is triggered from WiMAX to GSM grid, the GSM handover message is encapsulated in the container which is the element of WiMAX grid. The GSM handover message encapsulated in the WiMAX grid container looks exactly the same as it has been sent to GSM radio interface. On the other hand the mobile station consists of two separate and specific WiMAX and GSM parts. The GSM handover message encapsulated in WiMAX container will be received at the WiMAX specific part of the mobile station. Then this GSM handover message will be extracted here and passed to the GSM specific part of the mobile station as shown in Figure 6. As we know that WiMAX and GSM are different technologies, it is difficult to compare the results between these technologies. Hence, to overcome this problem, we calculate the results on the basis of threshold. Same procedure 


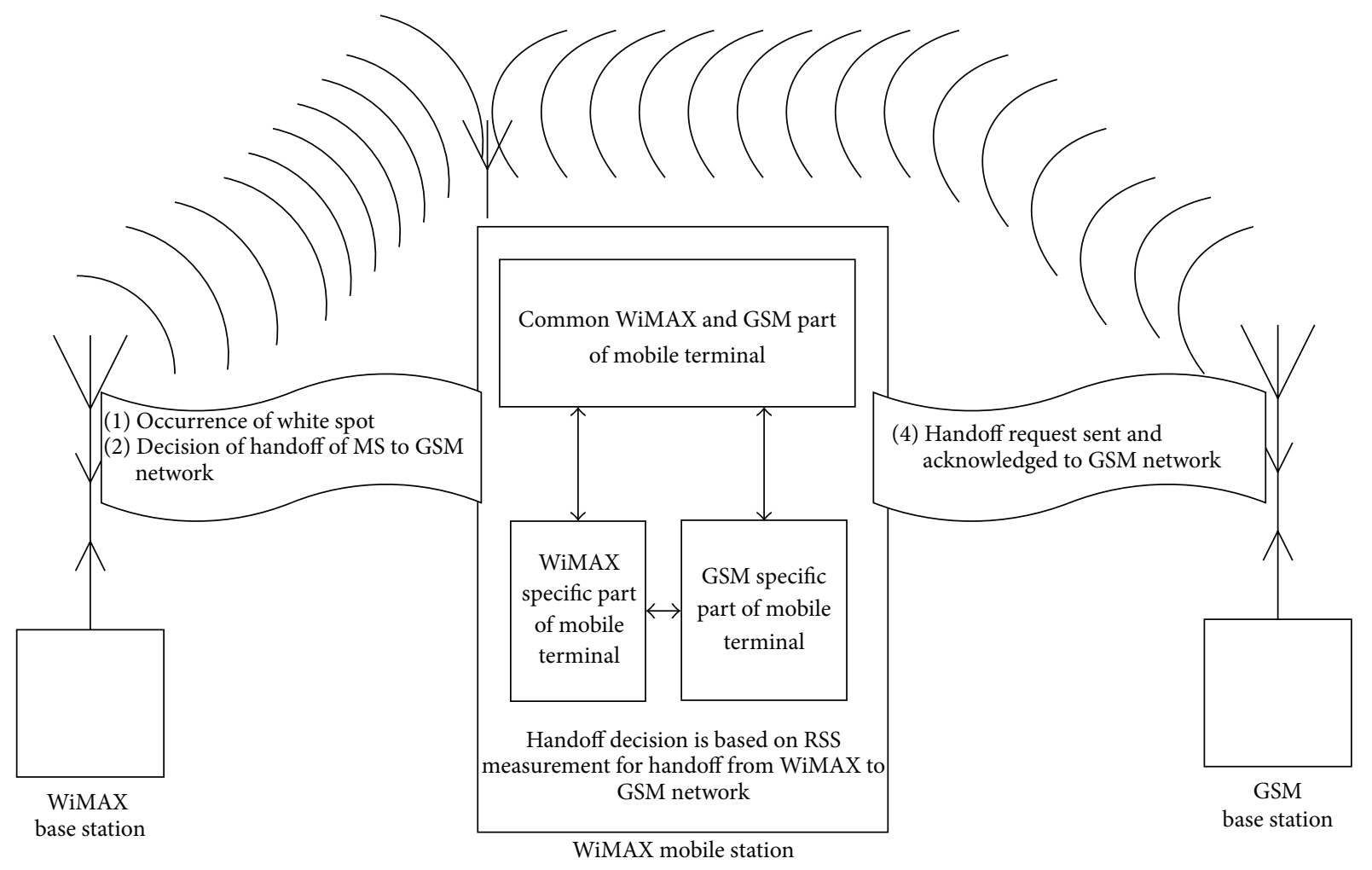

FIGURE 6: WiMAX to GSM handover.

of handoff is repeated when handoff is performed from GSM to WiMAX grid $[24,25]$.

In case of handoff from GSM to WiMAX grid, WiMAX handover message is encapsulated in the container which is the element of GSM grid. The WiMAX handover message encapsulated in GSM container will be received at the GSM specific part of the mobile station. Then this WiMAX handover message will be extracted here and passed to the WiMAX specific part of the mobile station as shown in Figure 7. The common part of WiMAX and GSM mobile terminal is a user interface LCD, which shows Received Signal Strength parameters of WiMAX and GSM grids [24, 25].

The cognitive mobile user is handed over to GSM base station present in the GSM grid, when white spot crops up and RSSI level declines below a certain threshold, that is, the minimum level required for retaining connectivity to WiMAX base station present in the WiMAX grid. The cognitive mobile user remains connected to base station of GSM grid unless the WiMAX grid base station is once more accessible for connectivity and is offering again good RSSI level for communication to WiMAX grid. In the scenario where there is no WiMAX base station present in nearby vicinity, the cognitive mobile user present in the GSM grid can even initiate horizontal handoff between GSM base stations unless WiMAX base station with good RSSI level is available within reach of the cognitive mobile user. When the desired appropriate threshold value in terms of RSSI level is sent to cognitive mobile user, then vertical handoff can be initiated back to WiMAX grid. WiMAX cognitive mobile user can initiate horizontal handoff between different WiMAX base stations present in the WiMAX grid on the foundation of good threshold value and RSSI levels [26].

5.3. Distance Formula. During the vertical and horizontal handoff, the MS moves along $x$ - and $y$-axis and changes its position. The distance formula is utilized to compute the gap between starting and ending point. If the specified points are $(x 1, y 1)$ and $(x 2, y 2)$, the distance between these points is specified by the distance formula as below [27]

$$
d=\sqrt{\left((x 2-x 1)^{2}+(y 2-y 1)^{2}\right)} .
$$

5.4. Received Signal Strength Formula. The Received Signal Strength is an essential parameter which helps to decide when to initiate handoff in order to avoid call drop and maintain connectivity [28]. It can be calculated as

$$
\mathrm{RSS}=10 \log (e) \times \log \left(\frac{1}{d^{2}}\right)=4.343 \times \log \left(\frac{1}{d^{2}}\right) .
$$

5.5. Radio Access Selection Formula. The Radio Access Selection (RAS) parameter decides to initiate handoff on the basis of throughput based on its Signal to Interference Noise Ratio (SINR). According to Shannon's formula, RAS is given as [29]

$$
\mathrm{RAS}=B \cdot \log 2(1+\operatorname{SINR}(j)),
$$




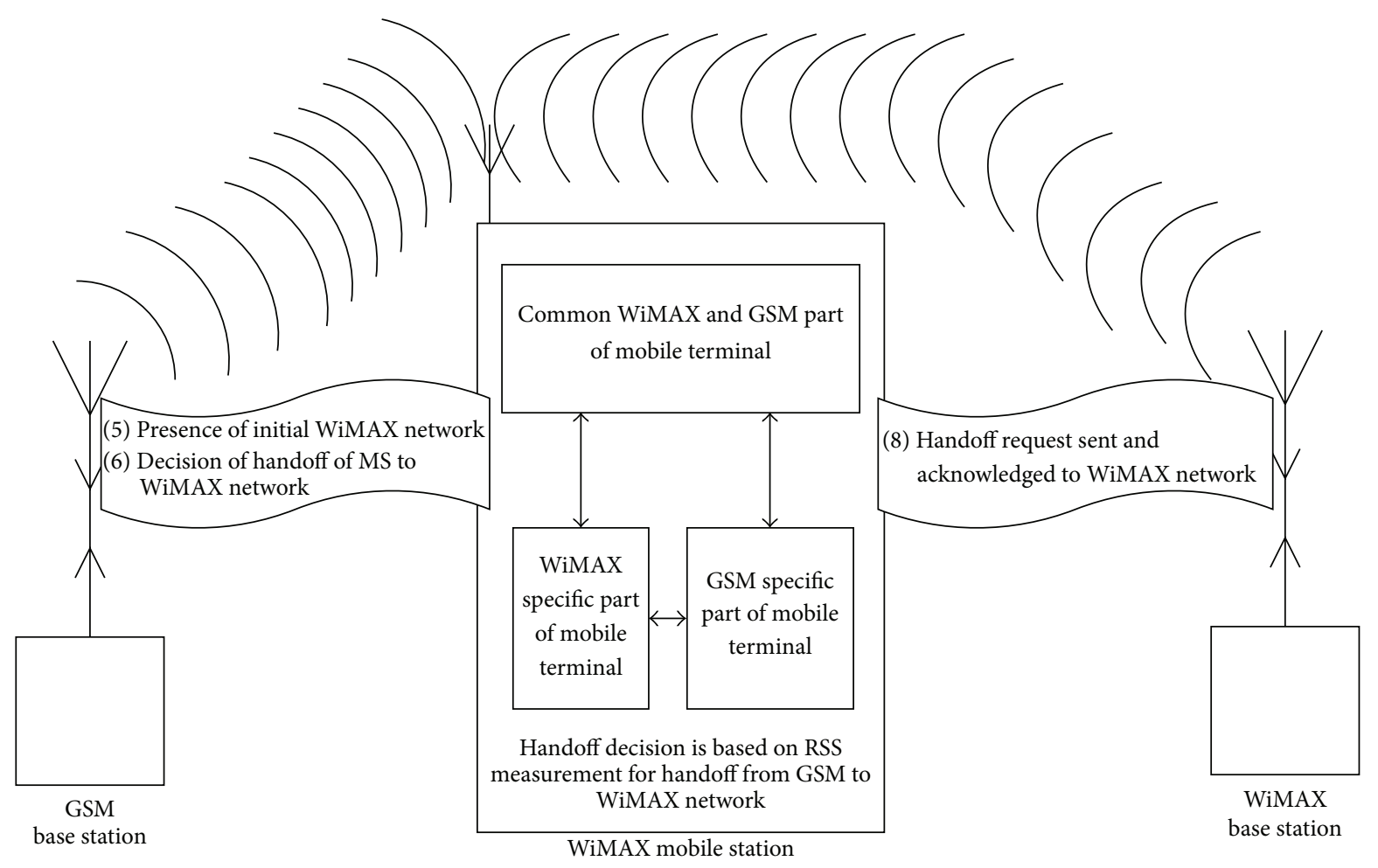

FIGURE 7: GSM to WiMAX handover.

where $B$ is the system bandwidth per radio access and $\operatorname{SINR}(j)$ is the signal to interference plus noise ratio practiced by mobile user in a particular radio access domain [29].

5.6. Channel Capacity Formula. We can also calculate WiMAX and GSM channel capacity, which tells us about the current condition of the channel and how much data rate can be supported by the channel [30]. It can be calculated as

$$
C=B \cdot \log 2(1+\mathrm{SNR}),
$$

where $B$ is channel bandwidth which is different for WiMAX and GSM channel and SNR is the ratio of communication signal to the Gaussian noise interference $[30,31]$.

5.7. Path Loss Formula. $\gamma$ known as path loss exponent relies on the transmission surroundings in which the signal is propagated. Propagation environment consists of the objects or obstacles present in the transmitter and receiver path [3133]. Path loss exponent can be calculated as

$$
\gamma=a-b \cdot h(\mathrm{BTS})+\frac{c}{h(\mathrm{BTS})},
$$

where $a, b$, and $c$ are terrain coefficients which depend on different terrain types $A, B$, and $C$. $h$ (BTS) represents the base station antenna height [32]. Similarly path loss for terrain type $C$ can be calculated as

$$
\mathrm{PL}=A+10 \gamma \log 10\left(\frac{d}{d 0}\right)+S,
$$

where $A$ is terrain coefficient for terrain $C, \gamma$ is known as path loss exponent, $d$ is the gap between transmitter and receiver equipment, and $S$ is the shadowing effect [31,34-36].

5.8. Free-Space Path Loss Formula. According to Free Space Path Loss (FSPL), the more far a receiving antenna from the transmitting antenna is, the less signal strength it will receive $[31,37]$. FSPL can be calculated as

$$
\mathrm{FSPL}=10 \log \left(\frac{\mathrm{Pt}}{\mathrm{Pr}}\right)
$$

where the transmitted power is $\mathrm{Pt}$ and received power is $\mathrm{Pr}$ [37].

5.9. Two-Ray Path Loss Model Formula. By using two-ray path loss model, the MS can easily estimate its distance from the neighboring base station to serving base station by measuring RSSI value. Two-ray path loss model can be calculated as [38]

$$
\operatorname{Pr}=\frac{(\mathrm{Pt} \cdot \mathrm{Gt} \cdot \mathrm{Gr} \cdot \mathrm{ht} \cdot \mathrm{hr})}{d^{4}},
$$

where Pr represents the received power which is to be measured from $\mathrm{Pt}$ (BS transmitting power). Gt is the antenna gain of transmitter, $\mathrm{Gr}$ is the antenna gain of receiver, ht is the transmitting antenna height, hr is the receiving antenna height, and $d$ is gap between the MS and neighboring base station $[38,39]$. 
TABLE 1: Range of input parameters of Hata model [38].

\begin{tabular}{lc}
\hline Carrier frequency & $1.5 \mathrm{GHz}$ to $2 \mathrm{GHz}$ \\
\hline Base antenna height & $30 \mathrm{~m}$ to $300 \mathrm{~m}$ \\
\hline Mobile antenna height & $1 \mathrm{~m}$ to $10 \mathrm{~m}$ \\
\hline Distance $d$ & $1 \mathrm{~km}$ to $20 \mathrm{~km}$ \\
\hline
\end{tabular}

TABLE 2: Range of input parameter for WiMAX cell radius [40].

\begin{tabular}{lc}
\hline WiMAX cell radius & Full featured \\
\hline LOS & 30 to $50 \mathrm{~km}$ \\
NLOS & 4 to $9 \mathrm{~km}$ \\
Indoor CPE & 1 to $2 \mathrm{~km}$ \\
\hline
\end{tabular}

5.10. Channel Modelling and WiMAX Cell Radius. In channel modelling, it is quite difficult to model the actual real time environment. Most of the simulation models are developed on the basis of empirical models. Empirical models use statistical tools to measure the broadcasting area of grid. By using empirical models, we cannot efficiently perform network planning of grid broadcasting areas, because empirical models are based on statistical probability. COST 231 extension to Hata model is the most suitable empirical model which can be utilized for handoff algorithm. According to IEEE 802.16e, Hata model operating frequency band is 2 to $6 \mathrm{GHz}$ within a cell radius of 1 to 3 miles and has mobility up to $120 \mathrm{~km} / \mathrm{h}$. For macrocell network planning environment, Hata path loss model is widely used for coverage calculation, where the base station antenna height must be above the rooftop level of the buildings adjacent to the base station $[40,41]$.

According to European Cooperative for Scientific and Technical (COST) research, the Hata model for $2 \mathrm{GHz}$ is given as [40]

$$
\begin{aligned}
& \text { PLurban }(d) \mathrm{dB} \\
& \begin{aligned}
= & 46.3+33.9 \log 10(\mathrm{fc})-13.82 \log 10(\mathrm{ht})-a(\mathrm{hr}) \\
& +(44.9-6.55 \log 10(\mathrm{ht})) \log 10(d)+\mathrm{CM},
\end{aligned}
\end{aligned}
$$

where $\mathrm{CM}$ is $0 \mathrm{~dB}$ for medium size cities and suburban areas and $3 \mathrm{~dB}$ for metropolitan areas $[40,42]$. The range of input parameters of Hata model is shown in Table 1. There are three categories of WiMAX cell such as interior self-installed Customer Premises Equipment (CPE), near line of sight (NLOS), and line of sight (LOS). The input parameters range of WiMAX cells with full featured configuration is shown in Table 2 [40].

\section{Simulation Results}

For implementation of our proposed model, we have used Microsoft Visual Studio, which provides a complete set of development tools for building XML web services, desktop applications, mobile applications, and ASP.NET Web applications [43]. The simulated topology for wireless grids based on cognitive heterogeneous method is equipped with 802.21 information server which has backhaul IP connectivity with two GSM and three WiMAX base stations. In the event of occurrence of white spots when RSSI value falls below the required threshold level for maintaining connectivity to WiMAX base station, the cognitive mobile user first initiates a vertical handoff to the GSM base station in order to avoid call drop. This vertical handoff of cognitive mobile user from WiMAX network base station to GSM network base station is imminent since there is no other WiMAX base station present in the nearby vicinity of cognitive mobile user. Once entered in the GSM network, the cognitive mobile user has to perform horizontal handoff from one GSM base station to another while remaining inside the GSM grid. When WiMAX grid base station is once again present in the vicinity of cognitive mobile user with the required RSSI threshold level for maintaining connectivity for an ongoing call, then vertical handoff will be executed by cognitive mobile user backward to the preliminary WiMAX grid. Once a cognitive mobile user is back in the preliminary WiMAX grid, it can now execute a horizontal handoff on the basis of required RSSI threshold level within the WiMAX grid from one WiMAX base station to another to maintain connectivity for an ongoing call. If a WiMAX mobile station user is currently connected to a WiMAX base station and the RSS value starts to fall below the threshold limit, then WiMAX handover process will trigger. The WiMAX mobile station will scan the neighboring WiMAX base stations and will decide to stay or to switch to another cell on the basis of RSS threshold limit. We have implemented the heterogeneous model for WiMAX 802.16e mobile user. The topology comprises three WiMAX base stations and two GSM base stations having backbone IP connectivity with 802.21 information server as shown in Figure 8. IEEE 802.21 standard provides media independent handover services and interoperability between different wireless and fixed grids. The simulation parameters being used for vertical and horizontal handoff have been shown in Figure 9. The input parameters based on WiMAX cell channel modeling being used for simulation are shown in Figure 10. For medium size cities and suburbs, the $\mathrm{CM}$ is $0 \mathrm{~dB}$ and, for metropolitan areas, it is $3 \mathrm{~dB}$ [40]. The Hata model is restricted to use the range of parameters being shown in Figure 10 [44]. WiMAX cell radius is categorized into three respective categories: line of sight (LoS), near line of sight (NLoS), and indoor selfinstalled Customer Premises Equipment (CPE) [45].

6.1. Mobile Assisted IEEE 802.21 Handoff Protocols. IEEE 802.21 is a media independent handover algorithm. IEEE 802.21 has both terminal initiated and grid initiated handover. In terminal initiated handover, the cognitive user terminal exchanges different protocols with IEEE 802.21 information server via the serving base station, whenever the problem of white spots occurs because handoff to neighboring grid base station becomes necessary. During the handoff process, the cognitive user terminal and IEEE 802.21 information server exchange different kinds of link and MIH events as shown in Figure 11.

6.2. Grid Assisted IEEE 802.21 Handoff Protocols. The grid assisted handoffs are initiated by grid and grid mainly controls the handoff procedure. Here the grid uses MIH events, 


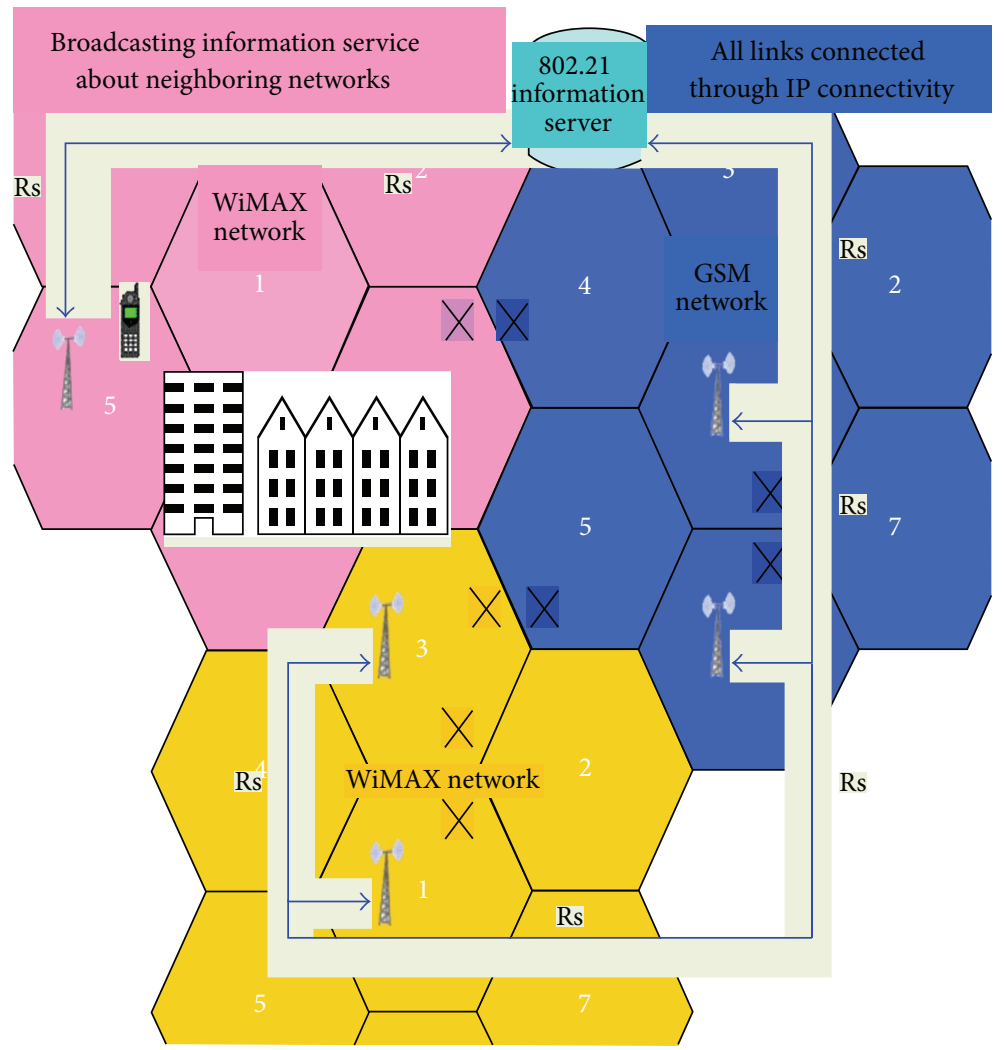

FIGURE 8: Simulation topology.

\begin{tabular}{|c|c|}
\hline Currert loction ol $M S(x y)$ & 327493 \\
\hline Distance BNBSS & $161 \mathrm{~m}$ \\
\hline Signal Stremgth & $44140 \mathrm{dm}$ \\
\hline Radodoccers Selection & $2549806 \mathrm{~Hz}$ \\
\hline Corsosver Locition $|x|)$ & 327416 \\
\hline Hysteesis Loction $|x|$ & 327471 \\
\hline WMASC Chamel Coposity & $770306 \mathrm{OHz}$ \\
\hline GSM Charnel Capacty & $746 \mathrm{~Hz}$ \\
\hline PathLoss Exponent & 5 \\
\hline Path Loss 1 [C] & 5008 \\
\hline Path Loss 2(C) & $105 \mathrm{de}$ \\
\hline FSPL & $468 \mathrm{~m}$ \\
\hline \begin{tabular}{|l} 
Two Pay Ground Model \\
\end{tabular} & $588 \mathrm{mi}$ \\
\hline
\end{tabular}

FIGURE 9: Simulation parameters.

\begin{tabular}{|c|c|}
\hline \multicolumn{2}{|c|}{ 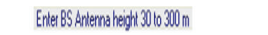 } \\
\hline BS Anterns hegint & $200 \mathrm{~m}$ \\
\hline \multicolumn{2}{|c|}{ 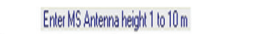 } \\
\hline MS Antemanh Heght & $2 m$ \\
\hline \multicolumn{2}{|c|}{ Enver Distare between } \\
\hline Didarced biwBS & $10 \mathrm{Km}$ \\
\hline \multicolumn{2}{|c|}{ 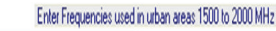 } \\
\hline 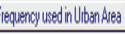 & $180 \mathrm{OH} \mathrm{HZ}$ \\
\hline ChamellModeling & 15208 \\
\hline \multicolumn{2}{|c|}{ 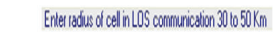 } \\
\hline Cellodids for LOS & $40 \mathrm{Km}$ \\
\hline \multicolumn{2}{|c|}{ 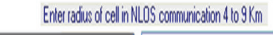 } \\
\hline Cellidadusfon NLOS & $5 \mathrm{Km}$ \\
\hline \multicolumn{2}{|c|}{ 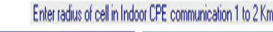 } \\
\hline 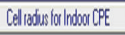 & $2 \mathrm{~km}$ \\
\hline
\end{tabular}

FIGURE 10: WiMAX system evaluation parameters. set of commands, and information from the information server to decide that handoff is required or not. If a vertical handoff is required, then the grid finds the target base station of an alternative grid and sends the command to cognitive mobile user to do the handoff to the desired base station. In grid assisted handoff, the grid controls all the steps of disconnection from the previous grid point of attachment to the new grid point of attachment. During the grid assisted handoff process, different MIH events which are exchanged between grid and IEEE 802.21 information server are shown in Figure 12 [26, 46].

6.3. Grid Access Security Steps. Whenever a cognitive mobile station has to connect to point of attachment in a grid, it has to pass through different grid access security steps, in order to authenticate itself. In step 1, the grid access authentication is 


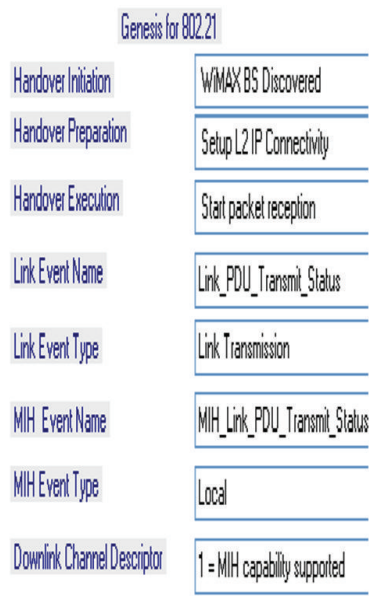

FIGURE 11: Mobile assisted IEEE 802.21 handoff protocols.

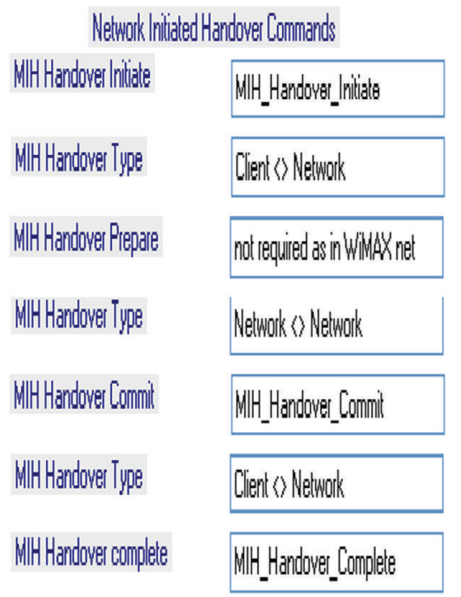

FIGURE 12: Network assisted IEEE 802.21 handoff protocols.

used to authenticate the cognitive mobile station to authenticate itself with the authentication server, before connecting to the point of attachment in the grid. In step 2, during secure association session, ciphering keys are used between the cognitive mobile station and authentication server. These keys are used to secure the link layer connectivity between the cognitive mobile station and authentication server. After successful grid access authentication and secure association, in step 3 (which is access control and ciphering), link layer data frames are exchanged between mobile node and point of attachment. Figure 13 shows the grid access security steps [47-49].

The decaying signal graphs due to white spot are shown in Figures 14(a) and 14(b). When Received Signal Strength starts decreasing and the connectivity of the mobile station with the current WiMAX base station starts to fail, it can result in the call drop if an alternative handoff is not initiated on time. The occurrence of the white spots is caused by the change in channel conditions which are sudden and cannot be predicted before time in real time wireless grid architecture.

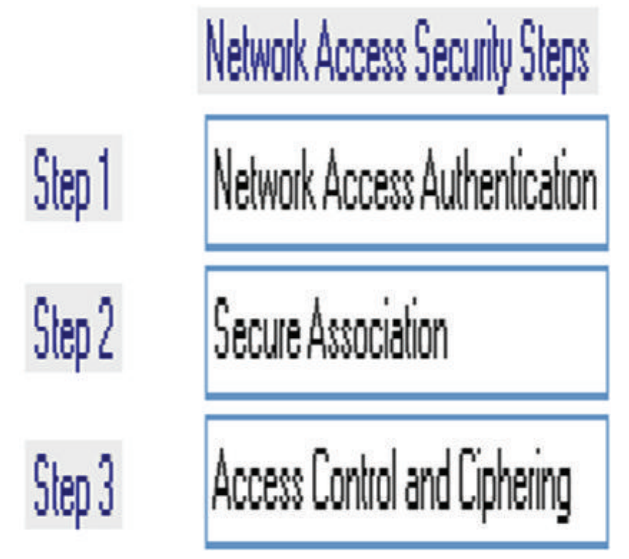

FIGURE 13: IEEE 802.21 network access security steps.

Figures 15(a) and 15(b) show the graphs of the growing signal of GSM grid, where a cognitive mobile user performs a vertical handoff to neighboring GSM grid base station because there is no alternative WiMAX base station available in the vicinity and horizontal handoff is impossible to perform. The other reason for this vertical handoff is the good Received Signal Strength of the GSM grid base station. Due to the occurrence of white spots, the signal starts to decay and MS interhandoff from WiMAX to GSM base station takes place in order to maintain connectivity during the call, on the basis of good RSSI values from the GSM base station. It has been depicted in Figure 16(a). Figure 16(b) shows an empirical model of signal strength of GSM grid with respect to the distance covered by mobile station towards the GSM base station, where a cognitive mobile user performs a vertical handoff to neighboring GSM grid base station as there is no alternative WiMAX base station available in the vicinity so horizontal handoff is impossible to perform. Another reason for this vertical handoff is the good Received Signal Strength of the GSM grid base station.

Figures 17(a) and 17(b) show the graphs of growing signal of WiMAX grid, where a cognitive mobile user performs a vertical handoff back to the initial WiMAX grid base station as initial WiMAX grid becomes available in the vicinity so no horizontal handoff is required within the GSM grid. Another reason for this vertical handoff back to initial WiMAX grid is the good Received Signal Strength of the WiMAX grid base station. In context to support the real time and killer multimedia applications, the cognitive mobile user will always prefer initial WiMAX grid over alternative GSM grid due to high data rate and greater broadcasting in near LoS communication.

Figure 18 shows the hysteresis margin, when a call drops due to white spot because the Received Signal Strength from serving base station falls below the required limit to main connectivity and therefore a handoff is required to the neighboring WiMAX base station for maintaining connectivity to the WiMAX grid. 
TABLE 3: RSS of WiMAX mobile station user with respect to WiMAX and GSM grids.

\begin{tabular}{lccl}
\hline Optimized time & $\begin{array}{c}\text { Current received } \\
\text { signal strength (RS) }\end{array}$ & $\begin{array}{c}\text { Radio access } \\
\text { selection }\end{array}$ & Current handoff status \\
\hline T0 = above threshold limit & $-29 \mathrm{dBm}>-31 \mathrm{dBm}$ & $1251 \mathrm{dBHz}$ & Currently in WiMAX grid \\
\hline $\begin{array}{l}T 1=\text { below threshold limit; } \\
\text { white spot occurred } \\
T 2=\text { searching neighboring } \\
\begin{array}{l}\text { WiMAX base station till } 20 \mathrm{~s} \\
\text { and failed }\end{array}\end{array}$ & $-31 \mathrm{dBm}<-30 \mathrm{dBm}$ & $600 \mathrm{dBHz}$ & $\begin{array}{l}\text { White spot has occurred and efforts to locate neighboring } \\
\text { WiMAX base station for horizontal handoff are } \\
\text { performed, but handoff fails as neighboring WiMAX base } \\
\text { station is not present }\end{array}$ \\
$\begin{array}{l}\text { T3 = vertical handoff to } \\
\text { GSM grid }\end{array}$ & $51.31 \mathrm{dBm}$ & $4500 \mathrm{dBHz}$ & $\begin{array}{l}\text { Neighboring GSM grid located with good RSS; now } \\
\text { vertical handoff to GSM base station is initiated }\end{array}$ \\
$\begin{array}{l}T 4=\text { horizontal handoff in } \\
\text { GSM grid }\end{array}$ & $44.46 \mathrm{dBm}$ & $\begin{array}{l}\text { As WiMAX grid is not located yet the mobile user can } \\
\text { initiate horizontal handoff within GSM grid to } \\
\text { neighboring GSM base station }\end{array}$ \\
$\begin{array}{l}T 5=\text { vertical handoff to } \\
\text { WiMAX grid }\end{array}$ & $47.9 \mathrm{dBm}$ & $1274 \mathrm{dBHz}$ & $\begin{array}{l}\text { Initial WiMAX grid located with good RSS; now vertical } \\
\text { handoff to initial WiMAX grid base station is initiated }\end{array}$ \\
\hline $\begin{array}{l}T 6=\text { horizontal handoff in } \\
\text { WiMAX grid }\end{array}$ & $44.14 \mathrm{dBm}$ & $\begin{array}{l}\text { A new WiMAX base station is present so mobile user can } \\
\text { initiate horizontal handoff within WiMAX grid to } \\
\text { neighboring WiMAX base station. }\end{array}$ \\
\hline
\end{tabular}

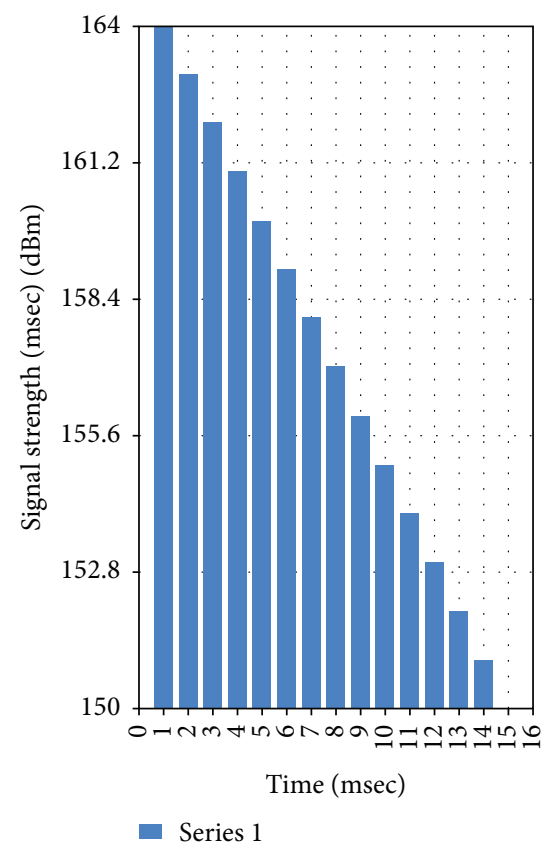

(a)

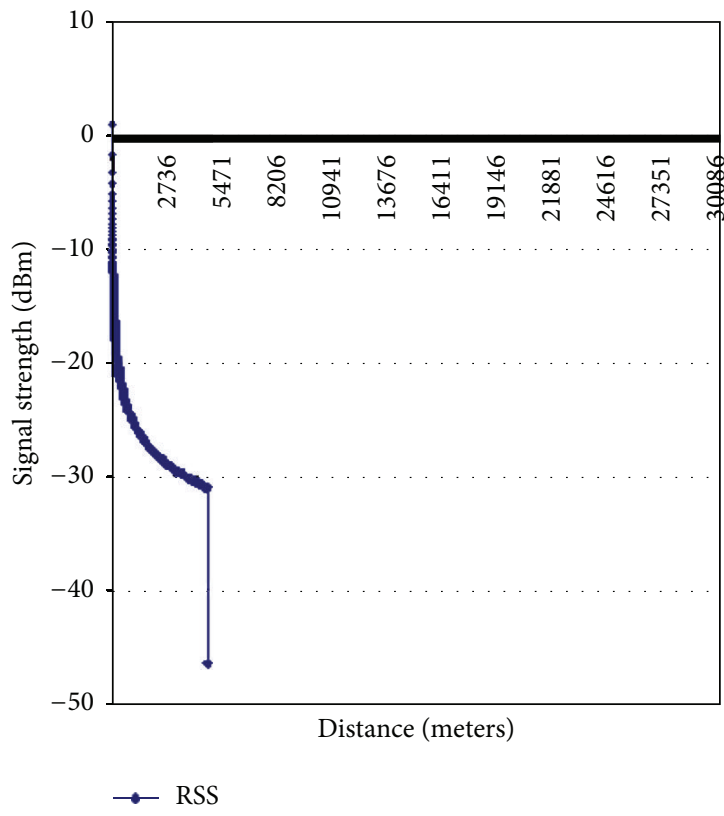

(b) RSS

FIGURE 14: Occurrence of white spot.

Table 3 shows the Received Signal Strength and optimized time of current handoff status of the mobile station user with respect to WiMAX and GSM base stations and scenarios such as horizontal and vertical handoffs within same grid or different wireless GSM and WiMAX grids.

\section{WiMAX and Its Relation to Internet of Things (IoT)}

First of all, we briefly talk about sensor network because it is the most essential component of Internet of Things (IoT).
Sensor network is basically a network of one or more sensor nodes, which communicate with each other through wired and wireless technologies [50, 51]. There are three main architectures of sensor networks found in the literature: flat architecture (in a multihop style, data is transferred from static sensor nodes to the sink node), two-layer architecture (to collect data from sensor nodes, more static and mobile sink nodes are deployed), and three-layer architecture (multiple sensor networks are connected together on the Internet). Therefore, IoT follows three-layer architecture [52]. We refer the readers to [53] where authors have listed 54 application 


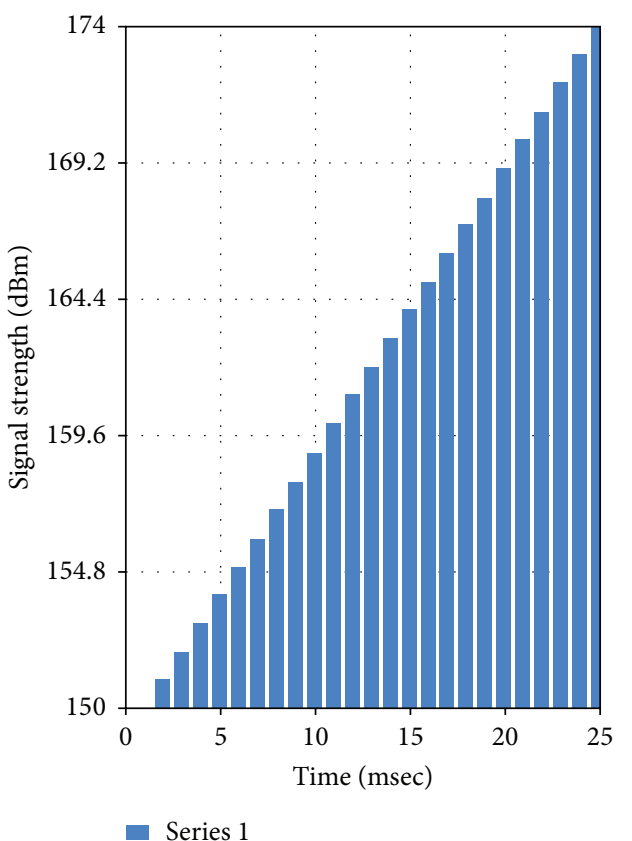

(a)

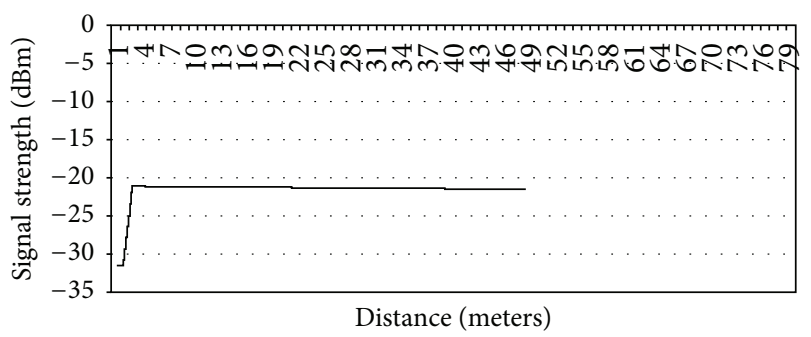

- RSS

(b) RSS

FIGURE 15: Handover to GSM network.

domains of sensor networks under twelve categories: smart cities, smart environment, smart water, smart metering, security and emergencies, retail, logistics, industrial control, smart agriculture, smart animal farming, domestic and home automation, and eHealth.

The concept of sensor network is existing long time before IoT was introduced. Hence, we can say that sensor network is not a concept that emerged with the IoT. However, the usage of sensor networks was very limited to achieve some specific purposes, such as environment monitoring [54], agriculture [55], medical care [56], event detection [57], and structural health monitoring [58]. Additionally, there are three categories of sensor networks that comprise the IoT [59]: Body Sensor Networks (BSN), Object Sensor Networks (OSN), and Environment Sensor Networks (ESN).

There are other technologies such as ad hoc networks that can also complement the sensing and communication infrastructure in IoT paradigm but these are clearly different from sensor networks and have many weaknesses. The differences are comprehensively discussed in [50]. Most of the sensors deployed today are wireless. There are several

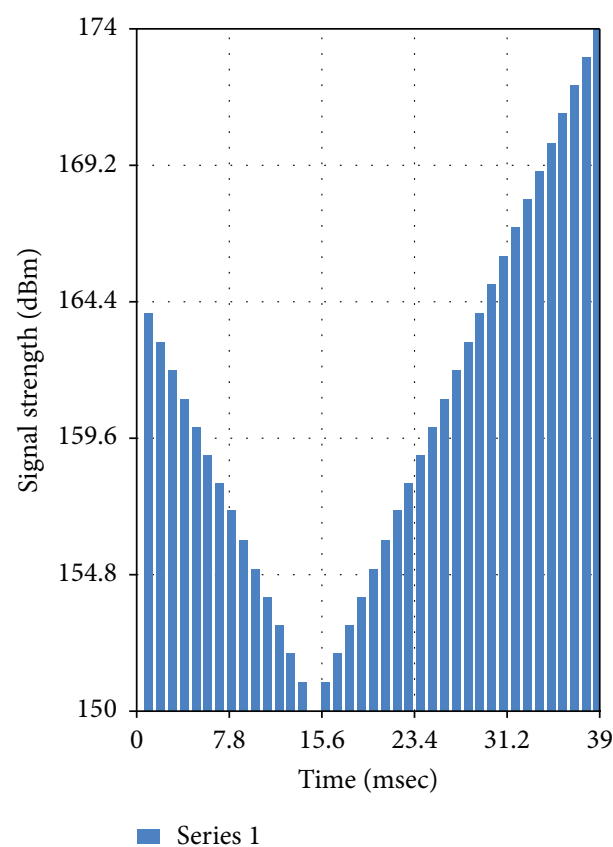

(a) Phenomenon of white spot and handover to neighboring GSM network

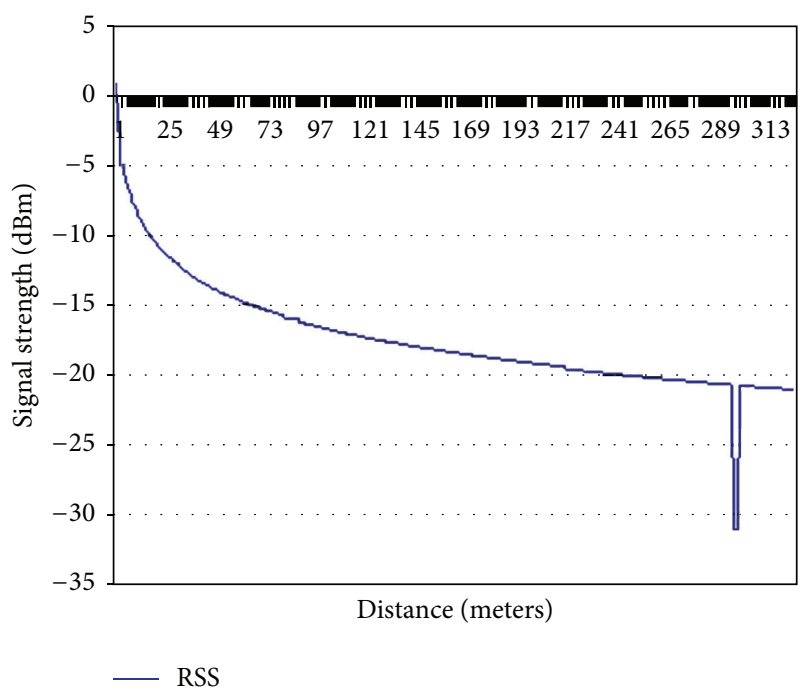

(b) RSS

FIGURE 16: Occurrence of white spot and handoff to GSM.

major wireless technologies used to build wireless sensor networks: wireless personal area network (WPAN) (e.g., Bluetooth), wireless local area network (WLAN) (e.g., WiFi), Wireless Metropolitan Area Network (WMAN) (e.g., WiMAX), wireless wide area network (WWAN) (e.g., $2 \mathrm{G}$ and $3 \mathrm{G}$ networks), and satellite network (e.g., GPS) [52].

Most of the sensors are deployed in a wireless fashion and WiMAX is a major wireless platform to provide cost effective multimedia services to the users. On the other side, IoT follows three-layer architecture of sensor networks. The research work carried out in this paper is vital to provide consistent and efficient service to a mobile user in the context of IoT paradigm. 


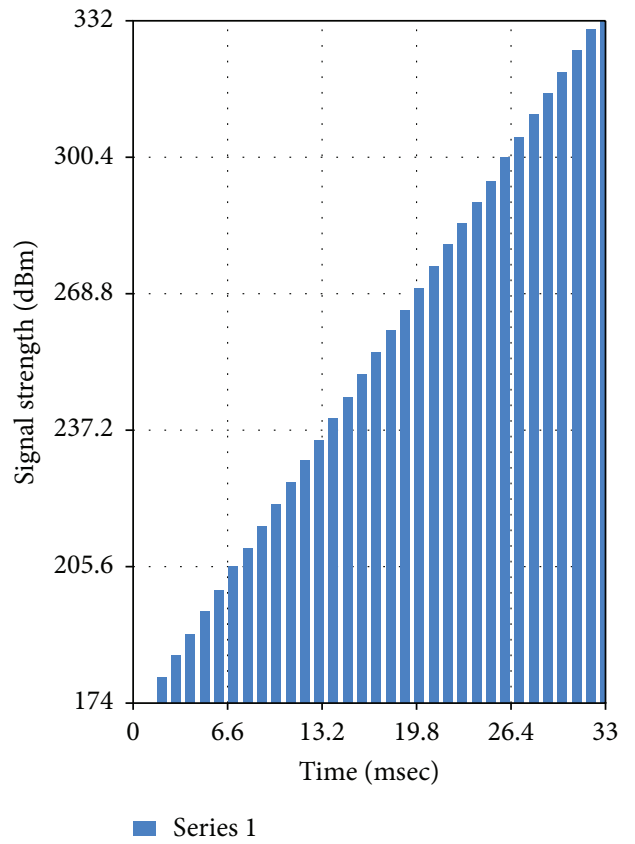

(a)

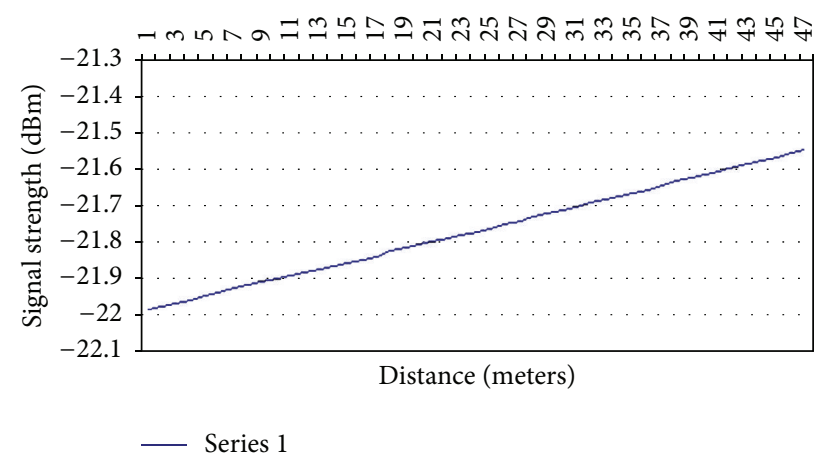

(b) RSS

FIgURE 17: Handoff to WiMAX network.

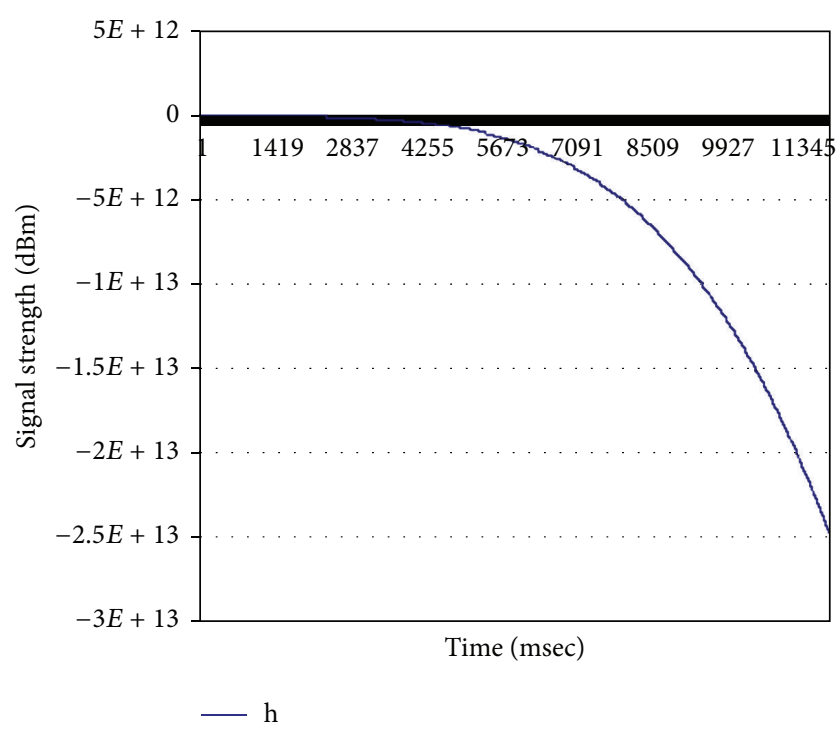

FIGURE 18: Hysteresis margin.

\section{Conclusion and Future Work}

The invention of wireless technologies in telecommunications industry has changed our lives and WiMAX is one of the leading technologies in the wireless domain, which has enormous prospects in future. In this paper, we have highlighted how white spot problem in broadcasting operation of WiMAX grids can be eliminated by utilizing horizontal and vertical handoff mechanisms in wireless grids based on the foundation of cognitive heterogeneous approaches. The proposed model based on horizontal and vertical handoff mechanisms is very cost effective in terms of providing economical real time services of voice, data, and streaming video to cognitive mobile subscribers.

Through detailed and comprehensive simulation experiments, we have shown how the problem of white spots can be avoided in different scenarios, that is, when a mobile subscriber switches from WiMAX base station to GSM and vice versa. We have clearly shown through simulation results how the signal grows from WiMAX/GSM grid, when a user performs a handoff in an efficient and timely manner.

The event of interhandoff state between two different technologies from WiMAX to satellite will be simulated in forthcoming research. The main intention for initiation of interhandoff between two different technologies from WiMAX to satellite by mobile subscriber is to retain connectivity in the event of occurrence of white spot, when there is no accessible opportunity for handoff to terrestrial wireless grid technologies.

\section{Abbreviations}

OFDMA: Orthogonal Frequency Division Multiplexing

SOFDMA: Scalable Orthogonal Frequency Division Multiplexing

MAN: Metropolitan Area Grid

GPS: Global Positioning System

GSM: Global System for Mobile

WLAN: Wireless Local Area Grid

RTPS: $\quad$ Real Time Polling Service

UGS: Unsolicited Grant Service

PSTN: Public Switched Telephone Network

BPSK: $\quad$ Binary Phase Shift Keying

QAM: Quadrature Amplitude Modulation

VoIP: $\quad$ Voice over IP

SLA: $\quad$ Service Level Agreement

NRTS: $\quad$ Nonreal Time Polling Service

FTP: $\quad$ File Transfer Protocol

AES: $\quad$ Advanced Encryption Standard

VLAN: Virtual Local Area Grid

3G: $\quad$ Third-Generation Mobile Technologies

QoS: $\quad$ Quality of Service

RSS: $\quad$ Received Signal Strength

WiMAX: Worldwide Interoperability for Microwave Access

IEEE: Institute of Electrical and Electronics Engineers. 


\section{Competing Interests}

The authors declare that there are no competing interests regarding the publication of this paper.

\section{References}

[1] A. Conti, O. Andrisano, B. Masini, and A. Bazzi, "Heterogeneous Wireless Communications for Vehicular Grids," Vehicular Grids TechniquesStandards and Applications, 2009.

[2] Westech Communications Inc, Can WiMAX Address Your Applications? WiMAX Forum, 2005.

[3] A. A. Minhas, A. Qaddus, and N. Rehmani, "Removal of white spots in WiMAX using cognitive heterogeneous wireless networks," in Proceedings of the 8th International Conference on High-Capacity Optical Networks and Emerging Technologies (HONET '11), pp. 99-105, Riyadh, Saudi Arabia, December 2011.

[4] D. Gray, "Mobile WiMAX-part I: a technical overview and performance evaluation," in Proceedings of the WiMAX Forum, August 2006.

[5] S. Bhunia, I. S. Misra, S. K. Sanyal, and A. Kundu, "Performance study of mobile WiMAX network with changing scenarios under different modulation and coding," International Journal of Communication Systems, vol. 24, no. 8, pp. 1087-1104, 2011.

[6] M. Riegel, D. Kroeseelberg, A. Chindapol, and D. Premec, Deploying Mobile WiMAX, John Wiley \& Sons, New York, NY, USA, 2011.

[7] C. Dou, "The maximum available radio resource of a WCDMA downlink," IEICE Transactions on Communications, vol. 88, no. 11, pp. 4309-4316, 2005.

[8] S. J. Vaughan-Nichols, "Achieving wireless broadband with WiMax," Computer, vol. 37, no. 6, pp. 10-13, 2004.

[9] A. Al Yusuf and M. Murshed, "Performance evaluation of multipath cellular networks in obstacle mobility model for downlink packet video communication," in Proceedings of the 68th Semi-Annual IEEE Vehicular Technology Conference (VTC '08), September 2008.

[10] G. Chadwani, S. N. Datta, and S. Chakrabarti, "Relay assisted cellular system for energy minimization," in Proceedings of the Annual IEEE India Conference (INDICON '10), October 2010.

[11] R. Jaichandran, A. Anthony Irudhayaraj, and J. Emerson Raja, "Effective strategies and optimal solutions for hot spot problem in wireless sensor grids (WSN)," in Proceedings of the 10th International Conference on Information Science, June 2010.

[12] K. Gierlowski, J. Woźniak, and K. Nowicki, "A hybrid-mesh solution for coverage issues in WiMAX metropolitan area networks," Journal of Telecommunications and Information Technology, vol. 2008, no. 1, pp. 1-24, 2008.

[13] R. Wahl, O. Stäbler, and G. Wölfle, "Propagation model and network simulator for stationary and nomadic WiMAX networks," in Proceedings of the IEEE 66th Vehicular Technology Conference (VTC-Fall '07), pp. 941-945, Baltimore, Md, USA, October 2007.

[14] G. Wolfle, "Propagation model and grid simulator for stationary and nomadic WiMAX grids," in Proceedings of the IEEE 66th Vehicular Technology Conference, October 2007.

[15] G. Zaggoulos, M. Tran, and A. Nix, "Mobile WiMAX System Performance-Simulated versus Experimental Results," 2008.

[16] A. Davydov, A. Papathanassiou, and A. Maltsev, "System level comparison of relay and RF repeater based technologies in WiMAX systems," in Proceedings of the IEEE Mobile WiMAX Symposium (MWS '09), pp. 205-208, Napa Valley, Calif, USA, July 2009.
[17] K. K. H. Kan, S. K. C. Chan, and J. K.-Y. Ng, "A dual-channel location estimation system for providing location services based on the GPS and GSM networks," in Proceedings of the 17th International Conference on Advanced Information Networking and Applications (AINA '03), IEEE Computer Society, CransMontana, Switzerland, July 2003.

[18] W.-F. Lu and M. Wu, "Analysis of communication traffic characteristics of two-hop-relay cellular system in the dead spots," in Proceedings of the 8th ACIS International Conference on Software Engineering, Artificial Intelligence, Networking, and Parallel/Distributed Computing (SNPD '07), vol. 2, pp. 540-545, IEEE, Qingdao, China, July-August 2007.

[19] N. Sultana, M. A. J. B. Bakkre, M. I. Islam, and M. R. Amin, "Performance evaluation of a mobile cellular network with two hop ad-hoc relaying," in Proceedings of the 12th International Conference on Computer and Information Technology (ICCIT '09), pp. 685-690, Dhaka, Bangladesh, December 2009.

[20] L. Jin, G. Chang, D. Jiang, and X. Wang, "Research on the discovery of blind spots in wireless sensor networks based on voronoi diagram," in Proceedings of the 9th International Conference on Hybrid Intelligent Systems (HIS '09), pp. 209-214, Shenyang, China, August 2009.

[21] S. McCann, "Next generation multimode terminals," 2008.

[22] International Engineering Consortium Conference, "Global system for mobile communication," in Proceedings of the International Engineering Consortium Conference, 2006.

[23] S. Ahmadi, "An overview of next-generation mobile WiMAX technology," IEEE Communications Magazine, vol. 47, no. 6, pp. 84-98, 2009.

[24] G. Alsenmyr, J. Bergström, M. Hagberg et al., "Handover between WCDMA and GSM," Ericsson Review, no. 1, pp. 6-11, 2003.

[25] A. Olsson, Understanding Changing Telecommunications: Building a Successful Telecom Business, John Wiley \& Sons, 2005.

[26] http://www.ieee802.org/21/Tutorials/802\%2021-IEEE-Tutorial .ppt.

[27] http://www.teacherschoice.com.au/maths_library/analytical\% 20geometry/alg_15.htm.

[28] R. K. Martin, A. S. King, R. W. Thomas, and J. Pennington, "Practical limits in RSS-based positioning," in Proceedings of the 36th IEEE International Conference on Acoustics, Speech, and Signal Processing (ICASSP '11), pp. 2488-2491, Prague, Czech Republic, May 2011.

[29] L. Jorguseski, R. Litjens, C. Zhiyi, and H. Nikookar, "Radio access selection in multi-radio access systems," in Proceedings of the 14th IEEE Symposium on Communications and Vehicular Technology in the Benelux, pp. 1-5, Delft, Netherlands, November 2007.

[30] http://www.vmsk.org/Shannon.pdf.

[31] L. Jorguseski, R. Litjens, C. Zhiyi, and H. Nikookar, "Radio access selection in multi-radio access systems," in Proceedings of the IEEE Symposium on Communications and Vehicular Technology in the Benelux, Delft, The Netherlands, November 2007.

[32] W. Afric, B. Zovko-Cihlar, and S. Grgic, "Methodology of path loss calculation using measurement results," in Proceedings of the 14th International Workshop on Systems, Signals and Image Processing and 6th EURASIP Conference Focused on Speech and Image Processing, Multimedia Communications and Services, pp. 257-260, IEEE, Maribor, Slovenia, June 2007. 
[33] S. Grgic, "Methodology of path loss calculation using measurement results," in Proceedings of the 14th International Workshop on Systems Signals and Image Processing and 6th EURASIP Conference focused on Speech and Image Processing Multimedia Communications and Services, June 2007.

[34] "Channel Models for Fixed Wireless Applications," Version 1.0, in IEEE, Croatia, July 2001.

[35] J. Zhou and J. K.-Y. Ng, "Using LDA method to provide mobile location estimation services within a cellular radio network," Journal of Computers, vol. 1, no. 7, pp. 41-50, 2006.

[36] J. Zhou, K. M.-K. Chu, and J. K.-Y. Ng, "A probabilistic approach to mobile location estimation within cellular networks," in Proceedings of the 15th IEEE International Conference on Embedded and Real-Time Computing Systems and Applications (RTCSA '09), pp. 341-348, IEEE, Beijing, China, August 2009.

[37] http://www.mike-willis.com/Tutorial/PF4.htm.

[38] S. K. Ray, S. K. Ray, K. Pawlikowskil, A. McInnes, and H. Sirisena, "Self-tracking mobile station controls its fast handover in mobile WiMAX," in Proceedings of the IEEE Wireless Communications and Networking Conference (WCNC '10), pp. 1-6, Sydney, Australia, April 2010.

[39] Y.-C. Chen, "An MAC protocol for wireless ad-hoc grids using smart antennas," in Proceedings of the 11th International Conference on Parallel and Distributed Systems (ICPADS '05), Fukuoka, Japan, July 2005.

[40] WiMAX Forum, WiMAX System Evaluation Methodology, Version 2.0, 2007.

[41] N. F. Rehmani, A. A. Minhas, and M. M. Alam, "Time domain efficient handoff scheme for mobile WiMAX," Australian Journal of Basic \& Applied Sciences, vol. 5, no. 8, pp. 1135-1150, 2011.

[42] A. Mourad and I. Gutierrez, "System level evaluation for WiMAX IEEE 802.16m," in Proceedings of the IEEE 28th International Performance Computing and Communications Conference (IPCCC '09), pp. 418-424, IEEE, Scottsdale, Ariz, USA, December 2009.

[43] http://www.msdn.microsoft.com.

[44] J. Cao, J. Liu, S. Zhu, and Y. Zhang, "A wide broadcasting wireless communication scheme for the intelligent distribution grid," in Proceedings of the World Congress on Information and Communication Technologies, Trivandrum, India, OctoberNovember 2012.

[45] E. Crozier and A. Klein, "WiMAX's technology for LOS and NLOS environments," in Proceedings of the WiMAX Forum, San Diego, Calif, USA, June 2013.

[46] S.-J. Yoo, "Predictive handover mechanism based on required time estimation in heterogeneous wireless grids," in Proceedings of the IEEE Military Communications Conference (MILCOM '08), San Diego, Calif, USA, November 2008.

[47] http://ieee802.org/21/Tutorials/802\%2021-IEEE-Security_Tutorial.ppt.

[48] C. Makaya, "Mobile virtual private grids: issues and challenges," in Emerging Wireless Grids Concepts Techniques and Applications, 2011.

[49] E. Qi, S. Bangolae, K. Sood, and J. Walker, "BSS transition optimizations and analysis for VoIP over WLAN," Wireless Personal Communications, vol. 43, no. 3, pp. 907-918, 2007.

[50] I. F. Akyildiz, W. Su, Y. Sankarasubramaniam, and E. Cayirci, "A survey on sensor networks," IEEE Communications Magazine, vol. 40, no. 8, pp. 102-114, 2002.
[51] M. Alnuaimi, F. Sallabi, and K. Shuaib, "A survey of wireless multimedia sensor networks challenges and solutions," in Proceedings of the International Conference on Innovations in Information Technology (IIT '11), pp. 191-196, IEEE, Abu Dhabi, The United Arab Emirates, April 2011.

[52] C. Perera, A. Zaslavsky, P. Christen, and D. Georgakopoulos, "Context aware computing for the internet of things: a survey," IEEE Communications Surveys \& Tutorials, vol. 16, no. 1, pp. 414-454, 2014.

[53] A. Asin and D. Gascon, "50 Sensor applications for a smarter world," Tech. Rep., Libelium Comunicaciones Distribuidas, Zaragoza, Spain, 2012, http://www.libelium.com/top_50_iot_sensor_applications_ranking/pdf.

[54] A. Mainwaring, D. Culler, J. Polastre, R. Szewczyk, and J. Anderson, "Wireless sensor networks for habitat monitoring," in Proceedings of the 1st ACM International Workshop on Wireless Sensor Networks and Applications (WSNA '02), pp. 88-97, ACM, New York, NY, USA, September 2002.

[55] J. Burrell, T. Brooke, and R. Beckwith, "Vineyard computing: sensor networks in agricultural production," IEEE Pervasive Computing, vol. 3, no. 1, pp. 38-45, 2004.

[56] D. Malan, T. Fulford-Jones, M. Welsh, and S. Moulton, "CodeBlue: an ad hoc sensor network infrastructure for emergency medical care," in Proceedings of the International Workshop on Wearable and Implantable Body Sensor Networks, 2004.

[57] S. Rooney, D. Bauer, and P. Scotton, "Techniques for integrating sensors into the enterprise network," IEEE Transactions on Network and Service Management, vol. 3, no. 1, pp. 43-52, 2006.

[58] A. R. Da Rocha, F. C. Delicato, J. N. de Souza, D. G. Gomes, and L. Pirmez, "A semantic middleware for autonomic wireless sensor networks," in Proceedings of the Workshop on Middleware for Ubiquitous and Pervasive Systems (WMUPS '09), pp. 19-25, ACM, Dublin, Ireland, June 2009.

[59] A. Gluhak and W. Schott, "A WSN system architecture to capture context information for beyond $3 \mathrm{G}$ communication systems," in Proceedings of the 3rd International Conference on Intelligent Sensors, Sensor Networks and Information (ISSNIP '07), pp. 49-54, IEEE, Melbourne, Australia, December 2007. 

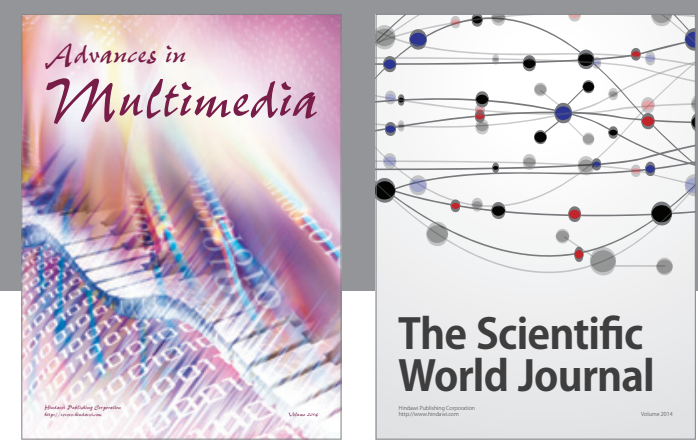

The Scientific World Journal
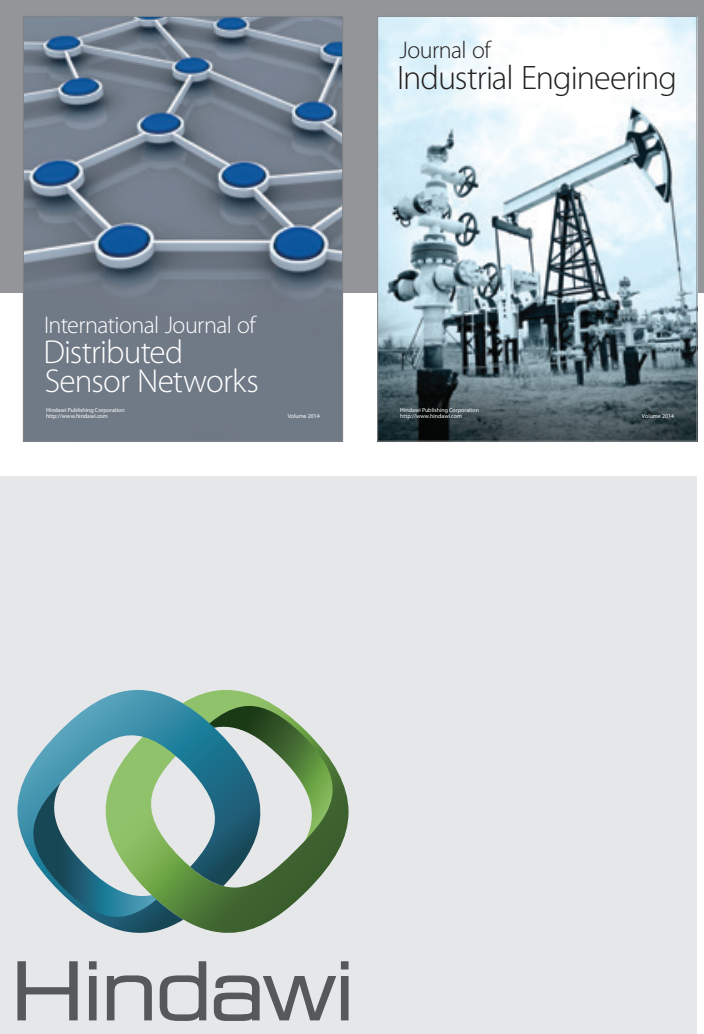

Submit your manuscripts at

http://www.hindawi.com

\section{Computer Networks} and Communications
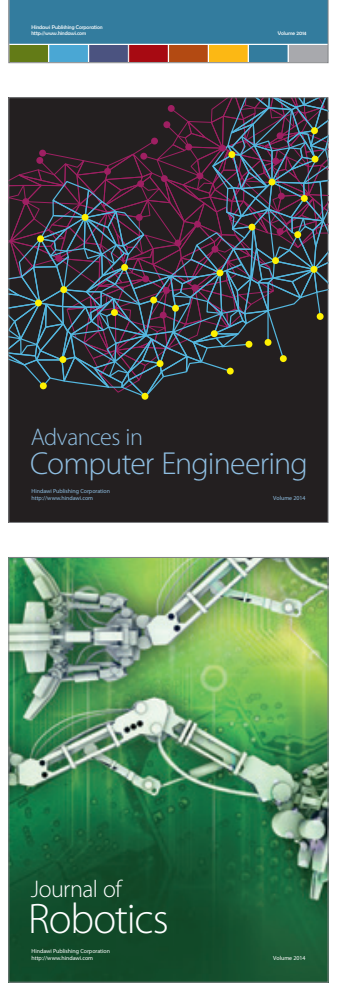
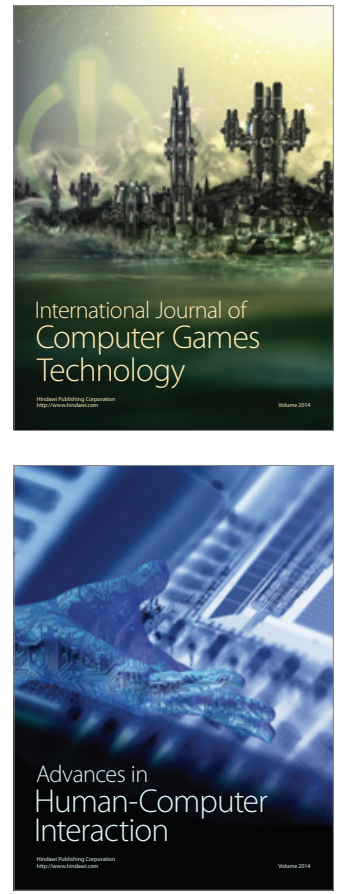
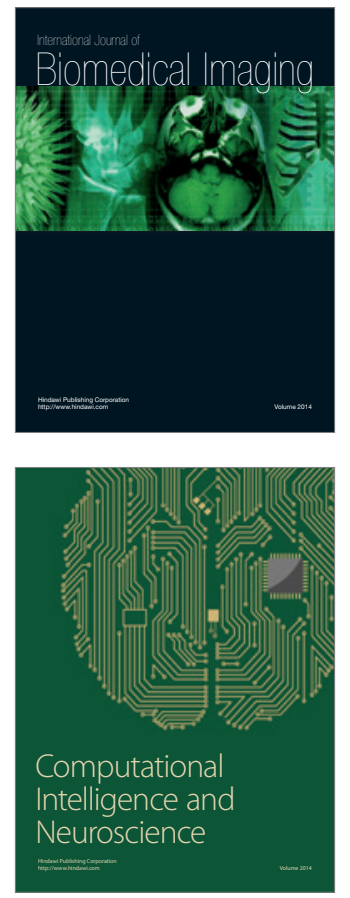
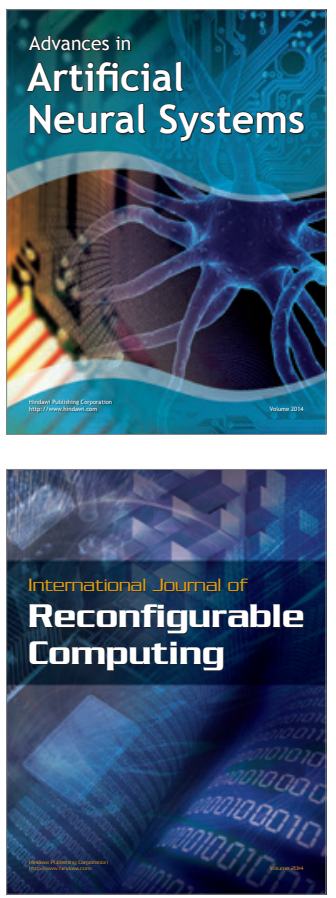
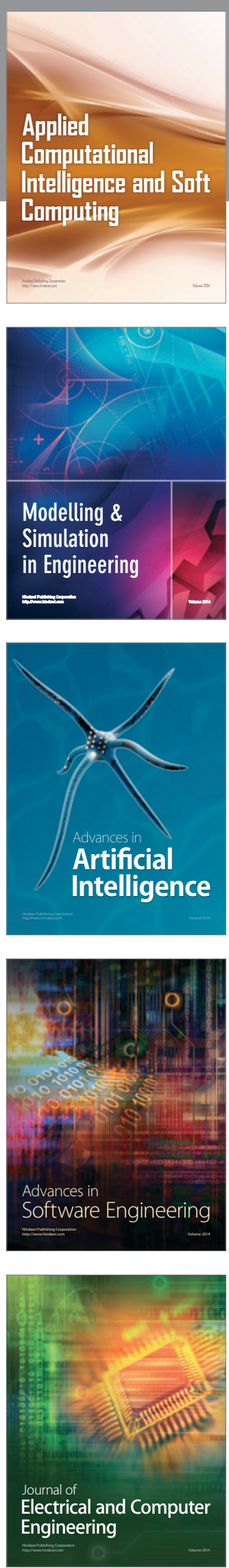